\title{
ENSEÑANZA EN LENGUAS REGIONALES Y SENTIMIENTO DE PERTENENCIA A LA ESCUELA
}

\section{SCHOOLING IN REGIONAL LANGUAGES AND SENSE OF BELONGING AT SCHOOL}

\author{
Julio Carabaña Morales \\ Mariano Fernández Enguita
}

\begin{abstract}
Sense of belonging at school is relevant both to academic results and to student wellbeing; it hardly depends on social and organizational factors, and it seems to be rather a personal trait. Nonetheless, it is in Spain clearly lower in bilingual regions, according to PISA data. In this paper we try to explain this difference. First we test the hypothesis of different languages being spoken at school and at home (school-home diglossia); empirical evidence forces us to reject it as well for PISA countries as for Spanish regions. Then we find that all students in schools using regional languages have lower scores in sense of belonging, with no influence whatsoever of their domestic language. We also find that regional language at school explains most of the differences among regions. Once these facts have been established, we advance some hypotheses in terms of schoolsociety diglossia: the sense of belonging at school suffers when the teaching language is the one of lower social preference or less social use.
\end{abstract}

Key words: sense of belonging, diglossia, bilingualism, school wellbeing, language immersion.

\section{RESUMEN}

El sentimiento de pertenencia a la escuela importa tanto para los resultados académicos como para el bienestar de los alumnos. Depende tan poco de variables sociales y organizativas que parece ser una característica puramente individual de los alumnos. Sin embargo, en España es notablemente menor en las CCAA bilingües, según los datos de PISA. En este artículo intentamos explicar esta diferencia. Verificamos primero la hipótesis de que se deba a la diglosia escuela-familia, con resultados negativos tanto a nivel de países como de las CCAA españolas. En el curso del análisis encontramos que son todos los alumnos que estudian en lenguas regionales los que deprimen el nivel de identificación con el centro, independientemente de cuál sea la lengua de su hogar. Encontramos también que esta variable explica la mayor parte de las diferencias entre las CCAA españolas. Establecidos estos hechos, avanzamos una hipótesis en términos de diglosia escuelasociedad: el sentimiento de pertenencia a la escuela padece cuando la lengua de enseñanza es la de menor preferencia o uso sociales.

Palabras clave: Sentido de pertenencia, diglosia, bilingüismo, bienestar escolar, inmersión lingüística

Fecha de recepción: 2 de octubre de 2019

Fecha de aceptación: 31 de octubre de 2019 


\section{INTRODUCCIÓN}

El sentido de pertenencia de los alumnos a sus escuelas (en adelante SdPE) es generalmente abordado como un factor relevante tanto para su bienestar inmediato como para sus resultados académicos.

Durante mucho tiempo la escolarización fue considerada exclusivamente un medio al servicio de fines más nobles y elevados. En términos meramente negativos apartaba a los menores de la ociosidad madre de todos los vicios y de la explotación laboral, simplemente de la calle o, más recientemente, de sexo, droga y rock-and-roll, asumiendo así una función de custodia, raramente mencionada por resultar poco glamorosa para los profesores, pero siempre esencial para las familias (¿qué haríamos si nos viésemos forzados a elegir entre seguridad y enseñanza para nuestros hijos?). En una perspectiva más positiva los preparaba para la incorporación a la vida adulta, a la economía, a la ciudadanía, y para alcanzar una mejor posición social (mejor, al menos, que sin ella). Se daba así por sentado que la escuela no podía ser muy gratificante, incluso que no tenía por qué serlo ni intentarlo, y se asumía y predicaba la asociación del éxito escolar a una moral victoriana, o de posposición de la gratificación, expresión de un carácter bien formado y propio de las clases medias.

Pero, desde finales del pasado siglo, esta visión ha entrado en crisis. En primer lugar, por la universalización de una enseñanza obligatoria u obligada cada vez más extensa. Los sistemas educativos del mundo desarrollado han pasado de escolarizar poco a muchos (ni siquiera a todos) y mucho a pocos a escolarizar mucho a todos. Hace apenas cincuenta años (los que median para nosotros hasta hoy) la obligatoriedad escolar era nominalmente de ocho años, en la realidad de seis, la enseñanza primaria $-\mathrm{y}$, para una importante minoría, inefectiva- $\mathrm{y}$, el acceso a la secundaria académica y más aún a la universidad, era para unos pocos. Hoy, en España, la escolaridad obligatoria abarca diez años, pero es prácticamente universal desde tres años antes (infantil de segundo ciclo) y durante, al menos, dos años más, sea por el objetivo de generalizar el acceso a un título postobligatorio -llamamos abandono prematuro a su no obtención, aunque ya no sea obligatoria- o porque el fracaso entraña fácilmente la repetición de uno o dos cursos. Dicho de otro modo, la enseñanza no universitaria abarca típicamente quince años o más (algo más con sólo que haya escolarización en la primera etapa de educación infantil, lo que ya es mayoritario, o alguna repetición de curso, lo que es muy frecuente). Demasiado tiempo en la vida de cualquier persona, y tanto más en su etapa más vulnerable, como para considerarlo simplemente un medio al servicio de la vida plena que ya vendrá después.

En segundo lugar, el lema que fue objeto de crítica o crónica por Goya, La letra con sangre entra, no es ya de recibo. Ni la letra lo justifica todo ni parece que entre mejor así. El aprendizaje, al fin y al cabo, no es el mero producto pasivo de la enseñanza (aunque no falte quien todavía lo cree), ni de su replicación interna por el alumno, sino que requiere su interés y compromiso, sea extrínseco o intrínseco. La pedagogía activa siempre reclamó, de un modo u otro, que la educación debía ser atractiva para el educando: es un motto recurrente que, desde la scholé griega, resurge en la Ca' Gioiosa de Vittorino da Feltre, la educación negativa de Rousseau, el Kindergarten de Fröebel, la mente absorbente de Montessori, etc. A ello se sumó pronto la Psicología, que en este siglo ha señalado el apoyo o la interferencia de los sentimientos y las emociones en los procesos de aprendizaje (Damasio, 2006; Dweck, 2008; Pekrun \& al., 2002; Mega \& al., 2014), rescatando así la relevancia, no ya en sí sino a efectos académicos, del bienestar del alumno. Todo ello ha contribuido a que el bienestar del alumno en la escuela, sus sentimientos respecto a ésta, etc. hayan sido objeto de mayor atención. Los largos años de presencia en las aulas no pueden ser ya meramente justificados por sus fines sino que merecen ser valorados en sí mismos (Jencks, 1972) o como base la formación en una ética del cuidado (Noddings, 1992, 2003). También en la investigación va ocupando mayor 
lugar el bienestar de los alumnos, sobre un fondo de preocupación generalizada por el aparentemente creciente desapego del alumnado respecto de la cultura escolar, tanto más en el contexto de economía de la atención configurado por la expansión de los mass media y la internet.

Pues bien, hay un sólido acuerdo interdisciplinar en que el sentido de pertenencia es un factor esencial del bienestar individual. Abraham Maslow (1943) ya señaló, en el tercer nivel de su pirámide o jerarquía de las necesidades, la necesidad de afiliación (affiliation, que bien puede traducirse también como pertenencia), fundada en gran parte en la aceptación social. Baumeister y Leary (1995) desarrollaron en general la hipótesis de la pertenencia, la idea de que un mínimo de relaciones humanas es esencial para la salud física y mental y para la motivación. Parece haber un amplio acuerdo sobre su relevancia en el ámbito escolar. Jules Henry $(1965,1972)$, en estudios inigualados sobre la subcultura juvenil, hizo énfasis en la importancia de la aceptación y la popularidad en la adolescencia. Harris (1989) ha sostenido incluso que los grupos de iguales sustituyen completamente a la familia como grupo de referencia, desplazando a la escuela las cuestiones clave de la identificación grupal.

Ahora bien, el hecho de que el SdPE pueda considerarse casi como un valor en sí por su relación con el bienestar del alumno, no implica despreciar la influencia favorable que parece tener sobre los resultados escolares, tanto cognitivos como conductuales. En Sociología, James Coleman, comparando las escuela secundarias norteamericanas públicas, privadas y católicas, registró lo que hoy llamaríamos el mayor valor añadido de estas últimas y lo atribuyó en gran medida al sentimiento compartido de comunidad, es decir, de pertenencia (Coleman, Hoffer \& Kilgore, 1982; Hoffer, Greeley \& Coleman, 1985; Coleman \& Hoffer, 1987), un argumento reiterado por Bryk, Lee y Holland (1993) y recogido por Robert Putnam en su análisis del declive del capital social (Putnam, 1995). La investigación psicológica es mucho más prolífica y abarca una multitud de conductas (Osterman, 2000; Allen y Bowles, 2013).

En lo que va de siglo, la OCDE, sobre todo a través de PISA, ha contribuido mucho a resaltar la importancia del SdPE. Los análisis de PISA están enfocados a las competencias de los alumnos, sobre las cuales le encuentran efectos menores que los de las variables socioeconómicas. Así, sobre las puntuaciones PISA, en 2003 se encuentra un efecto positivo que desaparece cuando se controlan las características del hogar (OECD, 2005: Tabla 5.21b); en 2012, el SdPE explica menos del 1\% de la varianza en la puntuación de matemáticas (OECD, 2013: Tabla III.2.9). Pero PISA también se detiene en la relación del SdPE con la satisfacción general de los alumnos con la vida. En 2015 encuentra una fuerte relación entre manifestar baja satisfacción con la vida (un nivel de 4 o menos en una escala del 0 al 10) con el sentimiento de ser un extraño en la escuela (OECD, 2017, III, 122). En síntesis, PISA declara en 2016 que "un sentido de pertenencia a la escuela importa tanto para el rendimiento escolar como para la satisfacción del adolescente con la vida" (OECD, 2017, III, 129, box).

En todo caso, la felicidad de los alumnos en la escuela es una cuestión de interés por sí misma y su presencia en la prueba de la OCDE tiene la virtud de permitir la comparación entre los sistemas educativos de distintos países y, en algunos casos, como el español, entre los de las distintas comunidades territoriales o regiones. El origen de este artículo está en la sorpresa que supuso, a raíz de un breve trabajo más general y sobre datos secundarios de uno de nosotros (Fernández Enguita, 2018: 152), topar con la notable diferencia entre Cataluña y el resto de España en el SdPE declarado por los alumnos. Sorpresa, además, no sólo por la magnitud sino tanto o más por el sentido, por ser Cataluña una región comparativamente rica, con una larga y apreciada tradición de innovación pedagógica y reforma escolar y siempre pronta a celebrar los éxitos de su subsistema escolar en sí mismo y por su alegada contribución a la cohesión social (Som Escola, L'escola no es toca!, 
Immersió $=$ Cohesió, etc.). En efecto, teniendo España en PISA 2015 el valor más alto de todos los países participantes $(0,47)$ en el índice de pertenencia a la escuela, el de Cataluña quedaba significativamente por debajo $(0,18)$. Comparando después los valores del índice por CCAA, encontramos que eran sistemáticamente menores en las bilingües que en las monolingües. Nuestra primera hipótesis fue la diglosia hogar-escuela (considerada en el plano individual, como vivencia del alumno cuya lengua doméstica es distinta de la escolar). Para su contraste, hubimos de tener en cuenta la investigación precedente sobre los determinantes del SdPE. Analizando los datos de PISA, encontramos que los datos obligaban a descartar la hipótesis de la diglosia escuela-familia pero encajaban muy bien con un hecho más simple, la escolarización en una lengua regional ${ }^{1}$. Quedamos muy sorprendidos del resultado, del que no encontramos precedentes ni empíricos ni teóricos. Hemos intentado interpretarlo proponiendo como hipótesis que pueda deberse a la diglosia escuela-sociedad propia de las regiones bilingües.

La estructura del artículo sigue la pauta convencional. Resumimos primero (1) lo que hemos leído sobre determinantes del SdPE y derivamos de ahí unas hipótesis; luego (2) presentamos los datos y métodos con que las contrastamos, presentamos (3) los resultados del análisis, intentamos interpretarlos (4) y sacamos algunas conclusiones (5).

\section{EL ESTADO DE LA CUESTIÓN}

¿Qué se sabe sobre las razones por las que varía el SdPE? Comencemos por notar que hay una notable diferencia entre la impresión que se obtiene de los estudios etnográficos y la que transmiten los estudios correlacionales. Los estudios etnográficos tienden a presentar un elevado malestar entre el alumnado, en particular en alumnos de clase obrera o de minorías étnicas (Jackson, 1968; Willis, 1978; Jenkins, 1983; Fernández Enguita, 1999; Feito, 1990), aunque también han detectado su extensión, mutatis mutandis, a los alumnos de clase media (Fernández Enguita et al., 2010). En cambio, los estudios correlacionales transmiten el resultado de que el malestar es más limitado y está poco influido por el entorno doméstico de los alumnos. Entre estos son numerosos los realizados por psicólogos, con muestras pequeñas, planteamientos complejos y atención centrada en otras variables psíquicas, solo a veces extendida a variables escolares. Podemos tomar como punto de partida la revisión de Osterman (2000), que incluye numerosos estudios sobre la importancia del SdPE, pero solo unos pocos sobre los factores que lo influyen; junto a algunos rasgos de la organización, el principal parece ser la buena relación con los profesores, un hallazgo confirmado por investigaciones posteriores: "De los distintos componentes del clima social de la escuela el apoyo del profesor es sin duda el más destacado", asegura Juvonen (2006: 658) recogiendo resultados de, entre muchos otros, Goodenow (1993) y Wentzel (1997). La última revisión que hemos encontrado es un metaanálisis de 51 estudios con un total de 67367 sujetos; concluye que el apoyo del profesor (no el de los compañeros) y características personales como la autoestima son los predictores más poderosos, sin apenas influencia de rasgos como género o etnia, ni tampoco de las actividades extracurriculares o de las normas de seguridad de las escuelas (Allen y otros, 2018).

Muy diferentes de estos son los estudios incluidos en los Informes PISA o realizados con datos PISA, con muestras amplias, procedimientos uniformes, menor complejidad de los planteamientos y mayor atención a variables sociales, tanto de la escuela como de la familia. Encuentran un malestar muy reducido y también poco ligado a variables sociales. Los datos de PISA 2000, analizados en un monográfico (Willms, 2003), revelaron que la desafección no dependía del tipo de sistema educativo, ni de los recursos escolares, ni estaba ligada con el absentismo, pero que hay mayores

1 Usamos el término 'regional' para referirnos a las lenguas co-oficiales en las diferentes CCAA. 
niveles de compromiso estudiantil en las escuelas con más disciplina, buenas relaciones entre alumnos y profesores y altas expectativas de éxito para los estudiantes. En PISA 2003, como promedio, para los países del área de la OCDE, el 81 por ciento de los alumnos está de acuerdo o muy de acuerdo con que su colegio es un lugar del cual sienten que forman parte. En la mitad de los países no hay diferencias por sexo, y en la otra mitad unas veces son favorables a los varones y otras a las mujeres. La relación con las puntuaciones PISA (vistas como causa, no como efecto) tiende a ser débil. En la mayoría de los países, las diferencias entre centros sirven para explicar tan sólo el 4 por ciento de la variación total en el SdPE. En algunos países el SdPE es menor en los programas de formación profesional y los orientados directamente al mercado laboral que en los programas académicos (OECD, 2005:129 ss). En PISA 2012, la intensidad del SdPE pareció haber retrocedido ligeramente en algunos países, y en cuanto a los factores de variación no aparece relación con el sexo, pero sí con el estatus socioeconómico (ESCS): los alumnos en el cuartil inferior de ESCS están 0,25 dt (desviación típica) más abajo en el índice de SdPE que los alumnos del cuartil superior del ESCS (OECD, 2013:Table III.2.3c). En PISA 2015, los valores del índice siguieron bajando (OECD, 2017: Figure III.7.1). En el conjunto de la OCDE, en 2003, un 7\% de los alumnos dijo sentirse como extraño en la escuela, 11\% en 2014 y 17\% en 2015. En cuanto a los factores de variación, el informe de 2015 atribuye una parte 'sustancial' al ESCS, dice de la disciplina que es un predictor 'fuerte' y constata que los alumnos que perciben apoyo de sus profesores muestran mayor sentido de pertenencia (OECD, 2017: Figure III.7.8). Quizás el análisis más comprensivo de los determinantes del SdPE sea el llevado a cabo por Chiu y otros (2016) en 41 países con datos PISA 2003. Encuentran que las variables de país (PIB per cápita, cultura jerárquica, cultura colectivista) explican un $2 \%$ de la varianza total en el índice de SdPE; que las características de las familias (inmigrante, lengua extranjera en casa, estatus socioeconómico, libros en casa, riqueza familiar y comunicación familiar) explican un 3\% adicional; que los rasgos de las escuelas (ESCS de los compañeros, relación alumno profesor, disciplina) explican un 7\% adicional y que las características de los alumnos (competencia lectora, autoeficacia, autoconcepto) explican tan solo un 1\% más. La variable más influyente de todas vuelve a ser la relación con los profesores.

En general, pues, según los informes PISA, en el conjunto de los países, particularmente de la OCDE, el SdPE depende algo del estatus familiar y del clima escolar, y muy poco de variables individuales. ¿Vale para España esta síntesis de los factores que influyen en el SdPE al nivel de la OCDE? Más bien no. En España, el índice de SdPE ha mejorado persistentemente. En 2000 (OECD, 2003) estaba en la media de la OCDE (que es cero, con dt 1); en 2003 alcanzó un valor de 0,20, es decir, un 20\% de dt por encima de la media de la OCDE; en 2012 subió otra vez a 0,41 y en 2015 volvió a subir a 0,47, situando a España a la cabeza de todos los países. En cuanto a los posibles determinantes, en 2003 hay diferencias significativas entre varones $(0,24)$ y mujeres $(0,16)$; la asociación con las puntuaciones PISA de Matemáticas es, medida con correlaciones, de 0,03 a nivel individual y de 0,13 a nivel de centro (OECD, 2005: Tabla 3.5c). En PISA 2012, los varones alcanzan 0,38 y las mujeres 0,44 , es decir, el incremento fue notablemente mayor entre las mujeres; y la correlación con la puntuación de PISA en Lectura es de 0,04 , menor que en el conjunto de la OCDE, 0,09. En PISA 2015, no hay diferencia por sexo, y la asociación con las puntuaciones PISA baja al 0,01, casi insignificante; hay también una ligera relación con el estatus del hogar: en la mitad inferior del ESCS, el índice de SdPE es de 0,40, en los cuartiles superiores de 0,50 y 0,58 (OECD, 2017: tablas III.7.8).

Es decir, si las variables familiares y escolares explican poca variación del SdPE en el conjunto de países de la OCDE, en España no explican prácticamente nada. No parece sino que el SdPE fuera una variable enteramente personal, que no sigue pautas sociales. Con este telón de fondo, resultan muy llamativas las diferencias que se observan entre Comunidades Autónomas. En el año 2003 se distinguieron por parte de PISA oficialmente solo tres CCAA, Castilla- León, Cataluña y País 
Vasco, pero Andalucía tuvo también su muestra propia. Como puede verse en la tabla 3, Cataluña está unos 0,37 puntos y el País Vasco unos 0,17 puntos por debajo de la media del 'resto' de España, diferencias ambas estadísticamente significativas. En el año 2012 quedaron sin muestra propia Canarias, Castilla la Mancha y Valencia. Como se ve en la tabla 3, en Baleares, Cataluña, Galicia, Navarra y País Vasco la media en el índice de SdPE es inferior a la de cualquiera de la demás CCAA, superando la diferencia entre las CCAA con lengua regional y el resto un tercio de dt. En el año 2015, las 17 CCAA tuvieron muestra propia en PISA. Pero este año no todas las CCAA con lengua regional puntúan igualmente por debajo del resto. Baleares, Cataluña y Galicia están 0,40 puntos por debajo, pero Navarra, el País Vasco y Valencia están solo 0,11 puntos por debajo, difiriendo más de las otras CCAA con lengua regional que del resto. Ocurrió que entre 2012 y 2015 el conjunto de los alumnos españoles incrementaron su puntuación en la escala de SdPE en 0,15 puntos (o dt), pero los alumnos de Navarra y la CAV lo hicieron en mayor medida, 0,23 puntos los primeros y 0,35 los segundos. Entre las demás CCAA el mayor incremento se dio en Castilla-León, con 0,25 puntos $^{2}$.

¿A qué puede deberse esta sorprendente diferencia entre CCAA, tan estrechamente relacionada con la existencia de lenguas regionales? A partir de los datos PISA se ha establecido una relación clara entre las puntuaciones en competencias y el hecho de que la lengua escolar sea distinta de la lengua doméstica (diglosia hogar-escuela) (Calero y Choi, 2019). Parece más obvio todavía que la diglosia influya negativamente en el SdPE a una escuela donde se habla una lengua distinta de la habitual en el hogar, tal como muestran los análisis de Chiu y otros $(2012,2017)$ para lenguas extranjeras. Como ya hemos dicho, el análisis mostró la conveniencia de sustituir esta hipótesis por otra más general. En fin, nuestra investigación puede verse como un contraste empírico de las siguientes hipótesis.

H1. Hipótesis tradicional sobre la diglosia: la diglosia Escuela-Familia (DEF) perjudica el sentido de pertenencia a la escuela en los países PISA.

H2. Hipótesis renovada sobre la diglosia: en España la diglosia Escuela -Sociedad (DES), la enseñanza en la lengua regional, perjudica el sentimiento de pertenencia a la escuela.

H3. En España, la enseñanza en lengua regional explica todas las diferencias en sentimiento de pertenencia a la escuela entre las CCAA.

\section{DATOS, VARIABLES, MÉTODOS.}

Si bien PISA incluyó la variable SdPE también en los años 2000, 2003 y 2012, limitamos el análisis a un año por brevedad y elegimos 2015 por incluir todas las CCAA. La muestra total es de más de 33000 casos, compuestos de una muestra nacional y de muestras complementarias para cada CCAA. Los casos se ponderan en relación al total español o en relación a cada CCAA según el marco de referencia del análisis.

El concepto de "sentimiento de pertenencia" es elusivo, y el término todavía lo es más (Slaten 2016). Para el ámbito escolar, Willms (2003: 8) lo ha cifrado en la aceptación por los pares y otros. Goodenow y Grady (2004: 60-61) lo definen como "la medida en que [los alumnos] se sienten personalmente aceptados, respetados, incluidos y apoyados por los otros -especialmente por los profesores y otros adultos en el entorno social de la escuela". El índice elaborado por PISA sigue

\footnotetext{
2 Estas diferencias en SdPE entre CCAA son todavía más sorprendentes si se tiene en cuenta que no se dan en la mayor parte de las numerosas variables personales que mide PISA, como motivación de logro, ansiedad ante las pruebas, gusto por la cooperación, valoración de la cooperación, etc. El único de estos rasgos en que las CCAA bilingües se diferencian algo del resto es la percepción de la justicia de los profesores.
} 
de cerca esta conceptualización. En una escala de Likert del 1 al 4 los estudiantes puntúan su sensación de ser o no marginados, si hacen fácilmente amigos, si se ven parte de la escuela, si se sienten fuera de lugar, si creen gustar a sus compañeros o si se sienten solos (pregunta ST034). Se construye con estos ítems un índice ( $\mathrm{SdPE}$ ) con media cero y desviación típica uno para el conjunto de la OCDE, de modo que las magnitudes quedan directamente expresadas en desviaciones típicas o size effects. (OECD, 2017, III, cap. 7).

En relación a la lengua, PISA informa de la lengua doméstica y de la lengua en que los alumnos han contestado el cuestionario y las pruebas, que por lo general es la de enseñanza de la escuela. Ahora bien, en la CA vasca no hay esta coincidencia, pues se deja a los alumnos elegir la lengua de la prueba; por ello, en esta CA la lengua escolar la obtenemos, no de las respuestas del alumno, sino a partir de los modelos de escolarización: al modelo D le atribuimos la lengua regional, a los modelos A y B la lengua nacional ${ }^{3}$.

La variable diglosia se construye a partir de la variable st022q01, que recoge si en casa se habla la lengua de la prueba u otra lengua. Se usa solo para la comparación con otros países en la tabla 1.

El resto de variables de lengua se construyen a partir de las variables langn, lengua hablada en casa, y langn-cog, lengua de la prueba. En la CA vasca, que deja a los alumnos elegir la lengua de la prueba, a todos los alumnos del modelo $\mathrm{D}$ se les atribuye el vascuence como lengua escolar. Estas variables son las siguientes:

Diglosi2: situación de diglosia (igual que 'diglosia', pero corrigiendo para la CA vasca).

Lengua escolar regional.

Lengua doméstica regional.

Español-Español, lengua española en casa y en la escuela.

Regional-Regional, lengua regional en casa y en la escuela.

Español-Regional, español en casa y lengua regional en la escuela.

Regional-Español, lengua regional en casa y español en la escuela.

Como variables de control se utilizan el tipo de gestión del centro (pública o privada), el Estatus socioeconómico del hogar (ESCS) y la puntuación PISA en Ciencias del alumno, aun cuando, como hemos visto, su influencia sobre el SdPE es más bien pequeña ${ }^{4}$.

La ecuación que estimamos con diversas especificaciones puede escribirse así:

(1) $\mathrm{SdPE}=a+b \mathrm{~T}+c \mathrm{~L}+\mathrm{dC}+\mathrm{e}$,

donde T denota variables territoriales (Comunidades Autónomas), L variables lingüísticas y C las variables de control.

\section{ANÁLISIS Y RESULTADOS}

\subsection{PERTENENCIA A LA ESCUELA Y DIGLOSIA EN LOS PAÍSES PISA}

Contrastamos en primer lugar la H1 (hipótesis de la diglosia escuela-familia) a nivel de país. Seleccionamos primero los países con más del 3\% de alumnos nativos cuya lengua doméstica

3 En el modelo A la lengua escolar es el español, en el modelo Del vascuence, en el modelo B ambas. Los alumnos del modelo B son pocos, y pueden fundirse con los del A sin sesgar los resultados.

4 No incluimos en el modelo otras variables individuales de motivación o actitudes, como podría ser el apoyo paterno, la más relacionada con el SdPE en la literatura, por la dificultad de dilucidar el sentido de su relación tanto con el SdPE como con las otras variables. Aun así, nos hemos cerciorado de que su inclusión no influye en los resultados. 
difiere de la escolar (variable st022q01 ta=diglosia), que son en total 31. Comprobamos que mientras estos alumnos tienen puntuaciones PISA en Ciencias inferiores en más de 0,20 DT en casi todos los países (menos Italia Luxemburgo, España y Túnez), son muy pocos los países donde estos alumnos se sienten menos parte de la escuela en la misma magnitud (Dinamarca, Estonia, Lituania, México, Perú, España y Uruguay). Así pues, a diferencia de lo que ocurre con las puntuaciones PISA, la hipótesis de la diglosia se cumple para el SdPE en tan pocos países que se los debe considera más la excepción que la regla.

¿Tienen estos países algo en común y distinto de los otros que pudiera explicar la peculiar relación entre diglosia y SdPE que se observa en ellos? Si separamos los países en que los alumnos diglósicos son mayoritarios (Luxemburgo), podemos dividir los restantes en dos grupos: uno en que la diglosia se debe a la presencia de inmigrantes que hablan lenguas diversas y otro en el que la diglosia se debe a la coexistencia histórica de diversas lenguas en el mismo territorio. Estos últimos son los que nos interesan aquí. Son solo 16 países, y están entre ellos seis de aquellos en los cuales los alumnos diglósicos se sienten menos parte de la escuela que los demás: Estonia, Lituania, México, Perú, España y Uruguay, es decir, todos menos Dinamarca. Puede, pues, decirse que la relación entre diglosia y SdPE se da sobre todo en países históricamente plurilingües, si bien no en todos.

Hay una amplia variedad en las relaciones entre lenguas en estos países. En México hay muy diversas lenguas indígenas (náhuatl, maya, zapoteco, etc.); en Perú también hay varias, pero predomina el quechua. En Uruguay la lengua minoritaria es una variedad del portugués llamada 'del Uruguay'. En estos países la lengua escolar es el español, de modo que solo hay un tipo de diglosia hogar-escuela, la de aquellos alumnos cuya lengua doméstica no es la oficial. En los países bálticos, el ruso es la lengua de una minoría nacional; en Lituania, además de la rusa, hay una minoría de lengua polaca. Si bien la mayor parte va a escuelas en ruso, otra está en situación de diglosia porque va a escuelas de lengua oficial. También en estos países hay un solo tipo de diglosia, aquel en el cual la lengua de la escuela es la oficial o mayoritaria. En esta situación, parece prudente sospechar que no es la diglosia por sí misma la que influye en el SdPE, sino las circunstancias que la acompañan, como, por ejemplo, la situación de los indígenas en México y Perú.

En España se da una situación más compleja, y excepcional tanto por el lado de las lenguas como por el lado de la organización política. Hay al menos tres lenguas, catalán, gallego y vasco, que se hablan además del castellano en diferentes partes del territorio nacional. En seis CCAA estas lenguas son co-oficiales y se usan en la enseñanza escolar, de modo que están en situación de diglosia tanto alumnos cuya lengua doméstica es la oficial del país, como alumnos cuya lengua doméstica es la cooficial de la CA. Hay incluso una CA, Cataluña, donde se obliga a todos los alumnos a escolarizarse en la lengua minoritaria, el catalán. Tanto esta complejidad como el hecho de que el tamaño de las muestras permita estudiarla a nivel regional hacen particularmente interesante el caso de España.

\subsection{PERTENENCIA A LA ESCUELA Y DIGLOSIA EN ESPAÑA}

Para reducirnos a los efectos de las lenguas autóctonas comenzamos separando los efectos de la inmigración y de las lenguas extranjeras. Chiu y otros (2012) han encontrado con datos de PISA 2003 de 41 países que los alumnos nativos tienen mayor SdPE que los alumnos inmigrantes y los que hablan una lengua extranjera en casa. Según se ve en la tabla 2, en España, la inmigración tiene efectos negativos sobre el SdPE de aproximadamente 0,30 puntos(o DT). Además son efectos acumulativos, aunque no perfectamente: los alumnos inmigrantes y diglósicos están unos 0,5 puntos más abajo en SdPE que los nativos monoglósicos. Al ser más frecuentes los alumnos diglósicos entre los inmigrantes que entre los nativos, su presencia infla un poco el efecto de la diglosia, que pasa de 0,31 puntos con ellos a 0,27 puntos sin ellos (tabla 2). 
Entre los nativos, parte de la diglosia se debe a que algunos hablan en casa lenguas no españolas. Aunque son muy pocos (en torno al 1\%) y su SdPE es solo un poco inferior al de los otros diglósicos nativos (tabla 2), los dejamos también fuera del análisis.

Las CCAA españolas han mostrado un gran interés por participar en PISA con muestra propia. En el año 2003 ya fueron cuatro las CCAA que lo hicieron, y en 2015 lo han hecho todas. ¿Cómo es a este nivel la relación entre diglosia y SdPE?

En primer lugar, se observa que las CCAA con situaciones de diglosia tienden a tener menor puntuación en SdPE que el resto. Como ya hemos hecho notar, en el año 2003 y en el año 2012 en todas las CCAA bilingües la media en el índice de SdPE es inferior a la de aquellas en que no se habla una lengua regional. En el año 2015 la divisoria es menos marcada, y mientras Baleares, Cataluña y Galicia están 0,40 puntos por debajo, Navarra, el País Vasco y Valencia están solo 0,11 puntos por debajo, difiriendo más de las otras CCAA con lengua regional que del resto.

$¿$ ¿Se debe esta diferencia entre CCAA con y sin lenguas regionales a la diglosia? Veamos si son efectivamente los alumnos cuya lengua doméstica no coincide con la de la escuela aquellos que rebajan las puntuaciones medias de las CCAA en la escala de SdPE. La tabla 4 deja ver la influencia de la diglosia en las seis CCAA donde la hay en 2015. En el conjunto de las seis, los alumnos diglósicos están 0,12 puntos (o dt) por debajo de los monoglósicos, una diferencia que, aun siendo estadísticamente significativa, es sustantivamente pequeña. Resulta, además, de situaciones dispares en las diversas CCAA. El efecto es apreciable solo en Galicia y Navarra, es nulo o pequeño en Baleares, Cataluña y Valencia y positivo incluso en la CAV.

Revisando sucesivamente las tablas 2 a 4, se ve que hemos avanzado un buen trecho en el análisis de la diferencia en SdPE entre alumnos en situación o no de diglosia que tomamos como punto de partida. Recordemos que en el conjunto de la población española es 0,36 puntos (o 0,36 dt) si usamos la variable PISA st022q01 (tabla 1), y de 0,34 si usamos directamente las variables de lengua, lo que va de 0,54 a 0,20 (tabla 2). Al quitar los inmigrantes, el SdPE de los diglósicos sube a 0,27, y al quitar los nativos con lengua extranjera en casa a 0,28 , dejando la diferencia en 0,26 puntos (O dt). Ahora bien, si en vez del conjunto de España consideramos solo las CCAA con lenguas regionales, el SdPE de los monoglósicos baja de 0,55 a 0,40 , dejando la diferencia en tan solo 0,12 puntos (o dt), que además es negativa y estadísticamente significativa solo en dos CCAA, Galicia y Navarra. Parece, por tanto, justificado alinear a España con los países en los cuales, en la tabla 1, dimos por despreciable la relación entre diglosia y SdPE, y rechazar, en consecuencia, la hipótesis (1) de la diglosia.

\subsection{PERTENENCIA A LA ESCUELA Y LENGUA ESCOLAR REGIONAL EN ESPAÑA}

No podemos decir que diferenciamos las cuatro combinaciones posibles de lenguas hogar-escuela inspirados por una teoría, o que fuera un caso de serendipity; se trató de pura curiosidad. Constituyó una gran sorpresa encontrar que como puede verse en la tabla 5, no es la diglosia escuela-familia, sino la escolarización en lengua regional la variable asociada con el bajo SdPE. Los alumnos escolarizados en lengua regional tienen un SdPE de 0,25 puntos (o dt), sin que su lengua doméstica suponga más que una diferencia despreciable. En cambio, los alumnos escolarizados en español tienen un SdPE por encima de los 0,60 puntos. En el panel b de la tabla 5 se hace el mismo cruce separando las CCAA bilingües. El valor del índice para los alumnos que usan el español en la casa y en la escuela es apenas dos centésimas menor que en las CCAA monolingües ${ }^{5}$. Encontramos,

\footnotetext{
${ }^{5}$ Resulta todavía más llamativo que cuando la lengua escolar es el español, los que usan en casa una lengua regional estén 0,10 puntos (DT) sobre los que usan la nacional, una diferencia pequeña pero estadísticamente significativa. Sugiere un cierto efecto para la diglosia, si bien en el sentido
} 
pues, diferencias de 0,35 puntos (o dt) asociadas no a la diglosia, sino al uso de lenguas regionales en la escuela.

Este inesperado hallazgo se ha formulado como segunda hipótesis, la que hemos llamado de la diglosia escuela-sociedad (DES), así que tiene poco de extraño que los datos la confirmen. Aunque volveremos sobre ella, podemos continuar con el contraste de la hipótesis 3, según la cual la escolarización en lengua regional basta para explicar las diferencias en SdPE entre CCAA. La regresión de la tabla 6 presenta una estimación de la ecuación (1) con los tipos de diglosia como variables determinantes. Contrasta la hipótesis de que se deben a la escolarización en lengua regional las diferencias en SdPE entre la España con y sin lenguas regionales (una versión reducida de la hipótesis (3)), utilizando como controles la gestión privada de los centros, el ESCS del hogar y la puntuación en PISA Ciencias del alumno.

Los resultados son concluyentes. En primer lugar, el déficit de 0,35 puntos en SdPE de las CCAA con lengua regional en la escuela parece deberse completamente a la escolarización en lengua regional. Más aún, no solo los alumnos escolarizados en castellano tienen en estas CCAA casi la misma puntuación en SdPE que en las demás, sino que aquellos cuya lengua doméstica es regional la tienen algo más alta (el coeficiente está al borde de la significatividad estadística al 5\%). En segundo lugar, la introducción de las variables de control no cambia la situación. La varianza explicada por cada grupo de variables puede tomarse como prueba de que estar escolarizado en lengua regional explica más varianza (cerca del $2 \%$ ) que la gestión privada del centro, el ESCS de la familia y la puntuación en PISA del alumno, que las tres juntas añaden tan solo un $0,5 \%{ }^{6}$.

Ahora bien, dado que este resultado es una media de las CCAA con lengua regional en la escuela, hay que preguntarse si se encuentra igual en todas o si hay diferencias entre ellas. El modelo (3) de la regresión en la tabla 6 parece indicar esto último, pues sustituyendo la variable que indica zona bilingüe por sendas dummies para cada CA, Baleares y Galicia aparecen con coeficientes en torno a los 0,15 puntos, y Cataluña de 0,11 . Puede tener interés, por tanto, comprobar el modelo para cada CA.

\subsection{PERTENENCIA A LA ESCUELA Y LENGUA ESCOLAR REGIONAL POR CCAA}

La tabla 7 presenta la frecuencia con que se da cada combinación de lengua hogar-escuela en cada CA con lengua regional (la ponderación es ahora la de cada CA, de ahí la diferencia de la media total). Cataluña es el caso extremo: el español no se usa en absoluto en la enseñanza, aun cuando es la lengua doméstica de más de la mitad de los alumnos. Pero Baleares no le anda muy lejos: del $47 \%$ de alumnos cuya lengua doméstica es el español, 8 están escolarizados en español y 39 en catalán, mientras que del 50\% que tienen como lengua doméstica el catalán, 46 está escolarizado en catalán y 4 en español. Navarra no se queda tan atrás. Un $8 \%$ de los alumnos tiene como lengua doméstica el euskera, de los cuales solo 3\% se escolarizan en esta lengua; los llamados a perpetuarla parecen más bien el 24\% de alumnos con lengua doméstica española escolarizados en vasco. En Galicia, el 60\% de la población usa español en casa, pero la mitad de ellos está escolarizada en gallego, mientras que solo 8\% usa gallego en casa y está escolarizado en español. Valencia es la única CA donde la situación aparece desequilibrada para los de lengua doméstica valenciana; son la cuarta parte y la mitad están escolarizados en español, mientras que los de lengua doméstica castellana son las tres cuartas partes y 10 puntos están escolarizados en valenciano.

opuesto al esperado: el mayor sentido de pertenencia lo manifiestan los alumnos de lengua doméstica regional que se escolarizan en la lengua nacional.

${ }^{6}$ Incluyendo en la estimación las variables de motivación y actitud construidas por PISA, tienen coeficientes beta significativos, en línea con investigaciones anteriores, la ansiedad ante las pruebas $(-0,09)$, la motivación de logro $(0,09)$, el gusto por la cooperación $(0,06)$, la valoración de la cooperación $(0,1)$ y el apoyo emocional de los padres $(0,14)$. En el conjunto de las CCAA bilingües, incrementan hasta el $8 \%$ la varianza de $2 \%$ explicada por la lengua escolar regional, pero no disminuyen el coeficiente de ésta. 
¿Se cumple la hipótesis 2 en cada una de estas CCAA tomadas por separado? En la tabla 8 se muestran los resultados de estimar el modelo 1 en cada una de ellas. Se toma como constante el índice de la situación español-español (en casa y en la escuela); los coeficientes expresan las diferencias con esta de cada una de las otras situaciones.

En Baleares, la media de los alumnos de referencia (EE) es 0,44, la de los alumnos que estudian en catalán es 0,23 puntos inferior si hablan español en casa (ER) y 0,18 puntos inferior si en casa hablan catalán (RR); por otra parte, la media de los alumnos escolarizados en español que hablan catalán en casa (RE) es 0,53, como si la escolarización en español mejorara particularmente el SdPE de los alumnos de lengua doméstica regional, pero es un efecto que desaparece cuando se controla el ESCS. No hay más que una manera de interpretar estos resultados: la escolarización en catalán afecta negativamente al sentimiento de pertenencia a la escuela.

En Galicia, la media de los alumnos de referencia (EE) es de 0,62, casi 2/3 de dt, igual que la media nacional. La de los alumnos escolarizados en gallego está 0,54 puntos por debajo si su lengua doméstica es el español y 0,49 si es el gallego (una diferencia despreciable). La combinación RE (gallego en casa, español en la escuela) está al mismo nivel que la EE. Sin lugar a dudas, la interpretación más sencilla vuelve a ser que la escolarización en gallego tiene efectos negativos sobre el SdPE, independientemente de si la lengua doméstica es gallego o castellano. Las variables de control, de las cuales la más influyente es el ESCS bajan considerablemente los coeficientes (en torno a 0,2 puntos), pero dejan igual las diferencias entre escuelas en castellano y escuelas en gallego. En relación al conjunto, Galicia es la región donde la lengua escolar tiene efectos más importantes, llegando a explicar el 4,5\% de la varianza total en SdPE, mientras las variables de control añaden menos del $1 \%$.

En Navarra las cifras también se ajustan sin roces a la hipótesis del efecto negativo de la lengua regional sobre el SdPE. La media de los alumnos de referencia (EE) es de 0,66 puntos, o 2/3 de $\mathrm{dt}$, mayor incluso que la media nacional; en cambio, si la lengua escolar es el vasco, el sentido de SdPE desciende en 0,45 puntos, igual si la lengua doméstica es vasco que si es español. Ahora bien, la situación RE está 0.18 puntos por debajo de la referencia, una magnitud importante aunque no llegue ser estadísticamente significativa; no cabe interpretarla como efecto negativo de la diglosia, pues los alumnos RR tienen resultados más bajos todavía, y los controles, de los que el único estadísticamente significativo vuelve ser el ESCS, la reducen a -0,11. También en Navarra, por tanto, se cumple la DES, o hipótesis (2) de la lengua regional. En relación al conjunto, en Navarra la lengua escolar tiene efectos solo menos importantes que en Galicia, pues explica casi el 3\% de la varianza total, más del triple de lo que añaden las variables de control.

En Valencia, los coeficientes son más complejos que en Baleares, Galicia y Navarra. En efecto, la puntuación media de los alumnos de referencia (EE) es de 0,575 puntos, cercana a la media nacional, y la escolarización en valenciano produce un efecto negativo de 0,43 puntos (cercano a los de Galicia o Navarra) en el caso de los alumnos con lengua doméstica castellana, pero solo de 0,22 (semejante al de las Baleares) en los alumnos con lengua doméstica valenciana, mientras que escolarizados en español estos últimos (RE) superan en 0,13 puntos a los alumnos de referencia (EE). Tomando como norma las situaciones monoglósicas, puede también decirse que la diglosia ER perjudica en 0,43 puntos, mientras que la diglosia RE favorece en 0,35 puntos. Ahora bien, no conviene quedarse con estos coeficientes, pues las variables de control los alteran notablemente. Tras ellas, lo parsimonioso es decir tan solo que la enseñanza en lengua regional empeora el SdPE de los que hablan español en casa (ER) en 0,2 puntos, despreciando que la diglosia RE lo mejora en 0,10 , dada la dudosa significatividad estadística de este coeficiente (16\%). En Valencia, por tanto, puede decirse tanto que ni la $\mathrm{H} 1$ ni la $\mathrm{H} 2$ se confirman como que ambas se confirman parcialmente, 
La clave de la dificultad parece estar en el coeficiente de 0,33 de los centros privados, que pudiera ser espurio y deberse a que no hay en ellos enseñanza en valenciano. Si dejamos solo los centros públicos (no mostrado), aparece un efecto de la enseñanza en valenciano en torno a -0,30 puntos para ambas lenguas domésticas, lo que confirmaría la DES también en Valencia. Pero el caso no es tan claro como los anteriores, y merecería más estudio.

Queda el País Vasco, que también se separa de la norma de Baleares, Galicia y Navarra. Los datos muestran un efecto negativo de la escolarización en lengua regional, pero, a diferencia de Valencia, limitado a los alumnos euskaldunes. Las variables de control (en realidad, el ESCS) disminuyen la constante, pero dejan prácticamente iguales los coeficientes. Llama la atención el poderoso efecto positivo de escolarizar en español a los alumnos euskaldunes, pero es un dato en el límite de la significatividad estadística, basado solo en unos 30 casos. Aun así, la conclusión, ciertamente paradójica, es que el modelo D de escolarización en vascuence daña la integración escolar de los alumnos euskaldunes. Como Valencia, el País Vasco es una media excepción a la DES, pero más interesante porque aquí es contraria a la hipótesis H1, o de la diglosia escuela-familia.

Hemos dejado para el final Cataluña, donde al enseñarse solo en catalán no es posible comparar con la enseñanza en español, ni por tanto contratar la hipótesis (2). La regresión, por tanto, solo muestra que los controles reducen a la nada el pequeño efecto ( 0,06 puntos, estadísticamente no significativa) negativo que se atribuiría a la diglosia desde los coeficientes brutos.

Resumiendo, hemos encontrado que la hipótesis de la diglosia escuela-sociedad (DES), que tan rotundamente se confirma en el conjunto de España, se confirma con menos claridad en dos de las cinco CCAA en que la hemos contrastado. Se confirma sin matices en Baleares, Navarra y Galicia, donde la enseñanza en lengua regional daña por igual a los de una u otra lengua doméstica; pero en Valencia daña solo a los de lengua doméstica castellana, y en el País Vasco a los de lengua doméstica vasca. En Cataluña no puede hacerse la comparación por falta de alumnos escolarizados en español.

Queda por comprobar qué ocurre con la hipótesis 3 cuando se la contrasta tomando por separado cada CA con lengua regional en lugar de con todas conjuntamente. La tabla 9 intenta sintetizar el análisis a nivel de CCAA en una única ecuación de regresión, siguiendo también el modelo de (1). Aparecen en primer lugar las diferencias entre CCAA que ya conocemos de la tabla 3: Baleares, Cataluña y Galicia tienen índices de pertenencia medios inferiores a los del resto. En el paso 2 se introduce, en vez de las tres variables de la tabla 7, una sola, la lengua regional en la escuela, cuya influencia es la que queremos contrastar; los coeficientes de las CCAA se reducen como prevé la hipótesis 3, pero no totalmente en Baleares y Galicia, que todavía quedan 0,10 puntos por debajo del resto de España, y en el País Vasco, que queda 0,13 puntos por encima. En el paso 3 introducimos variables que incorporan peculiaridades encontradas en las CCAA; dos para Galicia, donde tanto el efecto de ER como el de RR se han visto mayores que en el resto; una para la CAV, donde hemos visto solo hay efecto de RR y una para Valencia, donde hemos visto que solo hay efecto ER; se reducen a la nada los coeficientes de todas las comunidades, quedando solo el de Baleares, lo que confirma el acierto de las observaciones ad hoc. Finalmente, en el paso 4 introducimos los controles (centro de gestión privada, ESCS y puntuación PISA), que no alteran los resultados. La tabla 9 por un lado sintetiza los resultados a que hemos llegado en el análisis de cada CCAA, a los que debemos atenernos en caso de duda; y por otro confirma la hipótesis (3) en el sentido de que no todas las diferencias en sentido de pertenencia se deben a la enseñanza en lengua regional, pero sí la mayor parte. 
Resumamos, pues. Considerando en primer lugar España en su conjunto, hemos encontrado que la enseñanza en lenguas regionales tiene un efecto negativo sobre el sentido de pertenencia de los alumnos a los centros escolares; que se trata de un efecto sustancial, equivalente a un tercio de dt, mucho más fuerte que los de la gestión privada del centro, el ESCS doméstico y la puntuación PISA del alumno tomados en conjunto; y que basta por sí solo para explicar el menor SdPE observado en las CCAA con escuelas en lengua regional. Ahora bien, este resultado medio no vale igual para todas y cada una de las seis CCAA en las que hay escuelas en lengua regional. Tiene plena validez para Baleares, Navarra y Galicia, donde la enseñanza en lengua regional daña por igual a los de una u otra lengua doméstica; pero vale solo parcialmente para Valencia, donde solo daña a los de lengua doméstica castellana, y para el País Vasco, donde solo daña a los de lengua doméstica vasca. En Cataluña no puede hacerse la comparación por falta de alumnos escolarizados en la lengua nacional. Por último, la escolarización en lengua regional sería suficiente para explicar la mayor parte de las diferencias en SdPE entre CCAA, quedando pequeños residuos $(0,10 \mathrm{dt})$, negativos en Baleares y Galicia y positivos en el País Vasco.

\section{DISCUSIÓN}

\subsection{SOBRE EL CONCEPTO DE DIGLOSIA}

Wikipedia en español atribuye el concepto de diglosia, como es habitual, al lingüista Charles A. Ferguson, cuyo artículo del mismo título, Diglossia (Ferguson, 1959) encabeza cualquier búsqueda por ese término en Google Académico, con más de seis mil citas a día de hoy, más que triple lo que el segundo y treinta veces lo que el tercero. Ferguson, a su vez, se lo debe a Jean Psichari, filólogo francés de origen griego (aunque naciera en Odessa, entonces parte de Rusia y hoy de Ucrania), nacido Ioannis (o Yannis) Psycharis, y remite a un trabajo de éste publicado treinta años antes, "Un pays que ne veut pas de sa langue" (Psichari, 1928). Pero Psichari ya lo venía empleando desde 1886 (Mackridge, 2009), tomándolo del polígrafo Emmanuil Roídis (también transcrito Emmanuel, Emmanouil, Emmanouel, Roïdis, Rhoidis, Rhoidēs, y más recordado hoy, entre nosotros, por su obra La papisa Juana: un estudio sobre la Edad Media), que lo había estrenado en uno de sus textos recogidos en Apéndices (1885) y reiterado en Los ídolos (1893), para criticar el uso de dos variedades diferentes del griego (Katharevousa y Demotic, más o menos culto y popular), por los mismos hablantes, en distintas circunstancias. También puede encontrarse en H. Pernot (1902), precisamente en la introduccióna a una compilación de tres trabajos de Psichari.

La polémica griega versaba, pues, sobre dos variantes de la misma lengua. Tanto Roídis como Psichari, decididos demoticistas, criticaban no sólo el incómodo dualismo lingüístico en sí (diglosia, expresado desde sus raíces en griego), sino su artificialidad entre los hablantes de la versión culta (quienes manejaban de manera fluida la variante katherevousa también manejaban la demótica, aunque no a la inversa) y la incapacidad de esta para expresar diversos aspectos de la vida cotidiana y, en particular, los sentimientos, para su uso literario y sobre todo poético. Psichari consideraba la diglosia como un fenómeno anómalo en Europa, exclusivamente griego, debido al apego de las clases cultivadas por el pasado clásico perdido y a la influencia asiática, donde sí que podía encontrarse, por ejemplo, en las lenguas china y árabe.

Ferguson empleó el término en un sentido similar al de Roídis y Psichari: dos variantes dentro de una misma lengua. Pero lo que le dio más fuerza probablemente fue el hecho de subrayar la relación jerárquica entre ellas, denominando a las variantes como H (high) y L (low) - A y B (alta y baja) en español. Ferguson subrayaba la especialización de funciones (el uso en distintas situaciones) y su estabilidad multisecular y asociaba la primacía de la lengua $\mathrm{H} / \mathrm{A}$ a un prestigio, una tradición literaria, un nivel de normalización y una riqueza gramatical y léxica superiores. Y, ni lo último ni lo menos importante, una clara diferencia en el modo de adquisición: "los adultos invariablemente usan $\mathrm{B}$ al hablar a los niños y los niños usan $\mathrm{B}$ al hablar uno con otro. Como resultado, B se aprende invariablemente por lo niños en lo que puede considerarse la vía 'normal' de aprendizaje de la 
lengua materna de cada cual. A puede ser escuchada por los niños de vez en cuando, pero el aprendizaje de hecho de A se lleva a cabo sobre todo por medio de la educación formal. [...] Esta diferencia en el método de adquisición es muy importante. El hablante se encuentra cómodo con B en un grado que casi nunca alcanza con A.” (Ferguson, 1959: 331).

Posteriormente el concepto se vio expandido para abarcar la convivencia jerárquica de distintas lenguas en una misma población, así como el uso de códigos o registros distintos dentro de una misma lengua, aun sin el grado de diferenciación requerido por Ferguson. Aquí hay que mencionar especialmente a Joshua Fishman (1967), que distingue las cuatro combinaciones resultantes de cruzar las dimensiones de existencia o no de bilingüismo y de diglosia, y a John J. Gumperz (1962), quien amplía la cobertura del concepto para abarcar las situaciones con variedades más limitadas dentro de una misma lengua, con una diferenciación más funcional que estructural -a las que Ferguson había dejado fuera de la diglosia, a falta además de su significación histórica, para relegarlas a dialectos o registros dentro de una misma lengua. Este último criterio, más relajado, aunque ya no bajo el paraguas de la diglosia, llegará con fuerza a la sociología de la educación y la didáctica general transfigurado en los lenguajes público y formal, o los códigos restringido y elaborado, de Basil Bernstein, quien en la "Introducción" al primer volumen de Clase, Códigos y Control ya revelaba su estrecha vinculación con Gumperz (Bernstein, 1971). Sin embargo, tamaña expansión, una forma de morir de éxito, nunca fue del gusto de todos (Tollefson, 1983). Hudson, por ejemplo, considera que confunde más que aclara y aboga por volver al concepto de Ferguson, recordando que éste plantea como un elemento imprescindible que nadie o casi nadie hable la lengua o variante A en la vida cotidiana (Hudson, 2002).

En España, tuvo una notable aceptación la idea de la diglosia mientras sirvió para denunciar la posición subordinada de las otras lenguas que el castellano en sus distintos territorios de uso, principalmente por referencia a la noche del franquismo (Ninyoles, 1971; Siguán, 1976), si bien entre los lingüistas más militantes frente al predominio del español-castellano, y en particular en la lingüística catalanista, pronto se instaló la idea de que era más adecuado hablar de un conflicto lingüístico (Aracil, 1965; Ninyoles, 1971), conflicto que sólo podría resolverse con la normalización de la lengua subordinada o su sustitución por la lengua superordinada. La aplicación del concepto de diglosia a lenguas distintas, aunque puedan tener un tronco común (como catalán y castellano o gallego y castellano), o incluso no emparentadas (como vascuence y castellano) ya era en sí problemática, lo que llevó a algunos autores a hablar de biglosia (Fellman, 1975), diglosia intra o interlingüística (Pauwels, 1986) o pseudodiglosia (Hudson, 2002: 30, para el caso de catalán y castellano), pero, fuese cual fuese la diferencia léxica, morfológica, gramatical, fonética, etc. entre lenguas, dialectos o registros asumible, todas las variantes del concepto compartían y mantenían el atractivo de señalar su relación socialmente segmentada y jerárquica: A para la esfera pública y B para la privada. Pero en cuatro decenios de democracia (y, aunque sólo fuera a estos efectos, uno de dictablanda) ya ha llovido mucho: las lenguas de las nacionalidades, anfibológicamente llamadas propias (propio es antónimo de común, pero también de impropio y de ajeno) han sido, tanto en éstas como en otros territorios en los que eran de uso general (el catalán las Islas Baleares y la mayor parte de la Comunidad Valenciana) o simplemente relevante (el vascuence en parte de Navarra) (co)oficializadas a todos los efectos y positivamente discriminadas en las administraciones públicas y en la institución escolar para compensar su eventual vulnerabilidad en la sociedad civil y en la esfera de la cultura.

\subsection{ENTRE LA INMERSIÓN Y LA SUMERSIÓN LINGÜÍSTICAS}

El problema parece llegar cuando, en nombre de la compensación del predominio del castellano en los medios de comunicación, en la sociedad y/o por su dimensión transnacional, se opta no ya simplemente por el reforzamiento de la lengua propia en la escuela, como lengua vehicular, sino por su exclusividad, es decir, por la llamada inmersión lingüística, concepto en el que es imprescindible hacer un alto. La metáfora de la inmersión es intuitiva: tirar el niño al agua en vez de limitarse a 
explicarle cómo se nada, someterlo a una "exposición máxima”. En el ámbito del aprendizaje y la enseñanza de una segunda lengua (extranjera o, simplemente, distinta de la materna), significa sustituir su enseñanza como una asignatura más (por ejemplo, inglés, como si fuera, por ejemplo, geografía) por su uso vehicular en el aprendizaje de otras materias. La inmersión -en un contexto escolar- viene, al menos, del siglo XIV, cuando Vittorino Da Feltre la puso en práctica en su escuela Ca' Giocosa. Pero hay notables diferencias de grado: no es lo mismo enviar a un menor a un curso de verano en Irlanda que institucionalizarlo entre diez y quince años, en su propio país, en una segunda lengua como única vehicular. El ejemplo siempre aducido de inmersión lingüística es el de Canadá, o más concretamente Quebec, donde una parte importante de los alumnos que tienen por lengua materna el inglés son sumergidos por un tiempo en la enseñanza en francés. El sistema es flexible y, por tanto, admite múltiples variantes, pero en todo caso es voluntario (no obligatorio); comprende el uso de la segunda lengua como vehicular en varias asignaturas relevantes (no necesariamente en todas, sino entre el 50 y el 100\%); y es temporal (generalmente unos años de inmersión antes de desembocar en un sistema simplemente bilingüe o en la otra lengua). Los programas en los que la única lengua vehicular es la segunda, los profesores no entienden la primera y no incluyen apoyo a que los alumnos se expresen en ambas se denominan de sumersión o, de manera más rotunda, nada o búndete (sink-or-swim) (Swain, 2000; Cummins, 2009).

En España se denomina eufemísticamente políticas de inmersión a lo que en buena medida es sumersión. Lo es, en todo caso, porque se basan en que alguna lengua propia sea empleada como única lengua vehicular, tanto da que sea en todos los centros por imperativo político (que no legal, pues no lo han visto así los tribunales), como en Cataluña o (para la escuela pública) en las Islas Baleares, que sea para la mayoría de los centros en un contexto de elección familiar bajo presión social (como en el País Vasco) o que sea en un centro para el que no existen alternativas dentro de una zona escolar viable (como en áreas de Galicia, Navarra o la Comunidad Valenciana). En consecuencia, la condición de voluntariedad está ausente por completo en Cataluña, casi otro tanto en las Baleares, se ejerce bajo una notable presión social allá donde la política educativa de la administración educativa autonómica apuesta fuertemente por la inmersión, como es el caso del País Vasco, y puede tener importantes costes personales (distancia del domicilio, cambio de entorno social...) donde sencillamente surge de la opción mayoritaria de las familias de un centro, a su vez bajo la presión más o menos presente del profesorado, pero deja a los disidentes sin otras opciones de fácil acceso (lo que, por supuesto, también puede suceder al revés, en detrimento del uso vehicular de la lengua propia). En Quebec, la eterna referencia para la inmersión, ésta es voluntaria para los anglófonos, que siempre pueden evitarla si el niño, sus hermanos o sus padres acumulan cierto recorrido de escolarización en inglés.

Tampoco es, al menos cuando se realizan los planes de sus defensores, temporal (los centros de enseñanza secundaria dedicados de manera exclusiva o prioritaria a formación profesional tienen con mayor frecuencia el castellano como lengua vehicular en las comunidades autónomas con cooficialidad). No es simplemente una inmersión intensiva y prolongada, pero limitada, en la segunda lengua para evitar el uso de la primera como muleta y concentrar todo el esfuerzo comunicativo en aquélla (la "máxima exposición" es la justificación habitual de la inmersión). Es la exclusión definitiva e inapelable de la primera lengua como lengua vehicular, como instrumento del aprendizaje, como medio de comunicación en el contexto institucional. En definitiva, es la implementación deliberada de una relación de diglosia, a veces con el agravante añadido de que no se perpetra contra la primera lengua minoritaria sino contra la mayoritaria. Volviendo al manido ejemplo de Quebec, en un contexto de amplia autonomía de las autoridades locales y los centros la fórmula es variable, pero un formato típico a lo largo el ciclo K 12 sería, por ejemplo, tres años de inmersión total, tres o cuatro de semi-inmersión y cinco de seguimiento (Genesee \& al, 1986): en general se limita la inmersión el cien por cien del horario a pocos años y se transita después a la mitad aproximada de éste. 
A diferencia de otras situaciones de sumersión (por ejemplo, con los pueblos antiguos nativos en América, Oceanía y parte de África), el profesorado de cualquier comunidad autónoma en España sí que maneja de forma fluida y competente la lengua primera del alumnado (paradójicamente, es frecuente que también sea su primera lengua y que la maneje mejor que la de inmersión), y seguramente la utilizará en más de una ocasión, pero eso no quiere decir que se limite a utilizar la lengua propia de inmersión en el aula y menos aún a hacerlo en situaciones y de manera que pueda ser siempre entendida por los alumnos. Se puede apostar a que la sumersión es más relajada y permisiva en las primeras edades y se puede estar seguro de que varía por centros y por docentes, pero en todo caso pasará siempre por forzar el uso de la lengua regional hasta donde se considere, en la enseñanza propiamente dicha, y sabemos, asimismo, por las manifestaciones de no pocas administraciones, fuerzas políticas y asociaciones profesionales de corte nacionalista, así como por un inagotable rosario de anécdotas, testimonios y denuncias, que este uso se intenta llevar a menudo fuera de la enseñanza, del aula (a cualquier encuentro profesor-alumno, a las relaciones con los padres) e incluso de la relación profesor-alumno (a los espacios y tiempos de recreo). Por lo demás, hay que considerar no sólo la aptitud sino también la actitud del profesorado, y, por lo que sabemos, la inclinación nacionalista entre el mismo, sea cultural o política, es muy superior a la del conjunto de la población en cualquier comunidad autónoma (Fernández Enguita, 2011).

Si Psichari y Ferguson vieron la diglosia como una divisoria entre una minoría escolarizada en la lengua culta y una mayoría ajena a ella, hoy nos encontramos ante una nueva diglosia masiva alimentada y sostenida esencialmente por la escuela. Y si estos clásicos pudieron señalar la disociación que para la minoría culta suponía utilizar cada variante lingüística en un elenco distinto de situaciones y relaciones sociales, hoy vemos a la escuela universalizarla en ciertos contextos. En definitiva, podríamos estar asistiendo a una inversión de las viejas relaciones diglósicas por la que las lenguas antes en posición B, las lenguas propias, pasarían a situarse en posición A, mientras que la lengua común, el español, daría el salto contrario en las comunidades autónomas con lengua cooficial, aunque en distinto grado, y con distinto alcance social y territorial (Salvador, 1987; Álvarez, 1991; Blas Arroyo, 1999).

Volviendo a los datos que ahora nos ocupan, podría comprenderse desde esta inversión políticamente inducida de la relación que una experiencia de escolarización en lengua regional como única lengua vehicular, sin solución de continuidad, pueda no ser del agrado de los adolescentes, no sólo para aquellos que no la tienen como lengua primera o materna sino también para los que sí. Sabemos que, en oposición a la política escolar de inmersión, el castellano cuenta con la fuerza que le da ser la primera lengua de la mayoría y la lengua de comunicación de un colectivo más amplio: en el conjunto del territorio español y del territorio hispanohablante, como es obvio, e incluso en los territorios oficialmente bilingües. En el entorno digital, por añadidura, la implantación en el territorio inmediato de una lengua cualquiera pierde fuerza frente a la desterritorialización, o la transterritorialidad, de las lenguas más globales, como son la inglesa y, en un ámbito más restringido, la española. En consecuencia, los adolescentes (recuérdese que PISA examina a los alumnos de quince años) en régimen de inmersión/sumersión se ven forzados por la escuela a utilizar como única una lengua regional que de ninguna manera lo es (única) en su vida social y que puede no ser siquiera ni la más frecuente ni la de preferencia.

Esta tensión resulta omnipresente dondequiera que tenemos datos sobre los usos lingüísticos en el marco de una política de inmersión/sumersión. En el País Vasco, el informe Comportamiento lingüistico del alumnado, con datos de 2011 (Uranga, 2013) señalaba que el uso del vascuence es menor en el patio que en el aula, entre los alumnos que con el profesor y en la ESO que en Primaria. El elemento común es obvio: el castellano prospera fuera del entorno formal de la enseñanza, fuera del espacio reglamentado del aula, lejos de la autoridad del profesor y con el aumento en la capacidad y la voluntad de decidir libremente que trae la edad. En $4^{\circ}$ curso de Educación Primaria el $60 \%$ del alumnado habla entre sí sólo o más en vascuence que en castellano cuando está en el aula, pero, cuando sale al patio, el 59\% lo hace sólo o más en castellano: la proporción se invierte. 
En $2^{\mathrm{a}}$ curso de la ESO, los que se comunican con sus compañeros sólo o de manera preferente en castellano son ya el 60\%, y el patio el 76\%. (Uranga, 2013: 38-39).

En Cataluña el tema es también viejo. El uso del catalán ha sido impuesto de manera generalizada en las aulas, pero los patios son otro cantar. La recuperación o incluso el dominio del castellano en los patios aparece en Vila (2004), Galindo (2008), Bretxa y Vila (2013)... El catalán retrocede como lengua de elección de los alumnos en el paso de primaria a secundaria también en los medios de comunicación (Bretxa, 1016). Pero ninguna fuente como la Plataforma per la Llengua (PplL), "la ONG del catalán", que no hace mucho espió sin avisar los patios de las escuelas de Cataluña para registrar los usos lingüísticos de alumnos y profesores. De acuerdo con la Plataforma, apenas un $35 \%$ de las conversaciones entre alumnos en los patios de las escuela primarias se lleva a efecto en catalán, frente a un $65 \%$ en castellano; cuando se pasa a los centros de secundaria las proporcione son del 85 y el 15\%, respectivamente (PplL, 2019: 27). La ONG del catalán propone alguna explicación débil, como que los adolescentes crecidos eviten el catalán por asociarlo a la escuela primaria (tomada de Woolard, 2016), y alguna delirante, como que eviten el catalán por ser més elegant y elijan el castellano por ser més rude (PplL, 2019: 28). Al margen de estas minucias, en todo caso, queda el hecho de que, a pesar de la sumersión generalizada, el castellano se recupere en la comunicación entre iguales (alumno-alumno) frente a la comunicación jerárquica (alumnoprofesor), en los patios frente a las aulas y en la secundaria frente a la primaria.

Por la misma época en que Emmanouil Roídis tuvo la feliz idea de llamar diglosia a la dualidad dialectal y social de la lengua griega (1893), Ferdinand Tönnies tuvo la de señalar el tránsito de la comunidad a la sociedad (1887). Tan potentes ambas que los términos se han resistido a la traducción: diglosia se ha incorporado a todas las lenguas, al menos las de raíz grecolatina, y la dicotomía formada por comunidad y sociedad (o asociación) se acompaña habitualmente de los términos originales en alemán, Gemeinschaft und Gesellschaft. Sin entrar aquí en el alcance o los límites de una dicotomía tan aparentemente simple como la constituida por los tipos ideales de comunidad y sociedad (como status y contrato, tradición y modernidad, etc.), baste señalar que la comunidad estaría encarnada en figuras sociales relativamente pequeñas, espontáneas y orgánicas como la familia, el hogar, la aldea o la parroquia, mientras que la sociedad lo estaría en otras mayores, más racionales y artificiales como la ciudad, la empresa o el Estado. Parece obvio que el concepto de diglosia elaborado por Roídis y Psicharis y recogido por Ferguson y otros, apoyado en la oposición entre la lengua culta, escolar, etc. y la utilizada en la familia, el hogar, el vecindario, refiere a la disociación de la lengua escolar respecto de la comunidad. Lo que nosotros planteamos aquí, por el contrario, es su separación de la sociedad, es decir, de la gran ciudad, los medios de comunicación de masas y el entorno digital en los que viven los adolescentes, tanto si la lengua en ellos dominante es la de su hogar como si no. La diglosia siempre fue y sigue siendo un fenómeno social, aunque sentido como experiencia y vivencia individuales, pero los términos de la disociación y la imposición lingüística han cambiado y, los del malestar y la resistencia ante ellas, también.

\subsection{CONCLUSIONES}

Dado el enorme desarrollo de la investigación social, es cada vez más raro descubrir una regularidad empírica nueva. No pretendemos que la relación negativa entre aprender en lengua regional y sentimiento de pertenencia a la escuela sea una regularidad universal, pero pensamos que ha quedado bien establecida para España. Creemos que esta es la aportación principal de este trabajo.

Hemos seguido primero, en el estudio de la diglosia, las reglas del paradigma hipotético-deductivo y luego, en el de la lengua local, las del inductivo. En su estudio sobre la Lógica de las Ciencias Sociales, Habermas (2006) discutió razonablemente la complementariedad entre ambos modos de acercarse a la realidad social. Las teorías y las deducciones, basadas en la comprensión, deben ser contrastadas empíricamente. Es lo que hemos hecho, con resultados negativos, para la diglosia. Y las regularidades observadas por inducción, deben ser comprendidas. Hemos encontrado que la 
enseñanza en lenguas regionales tiene una relación negativa con el SdPE, pero nos parece estar lejos de comprenderla

Comprendíamos bien, a priori, cómo la diglosia hogar-escuela podría perjudicar el sentido de pertenencia a la escuela, especialmente cuando está asociada con un estatus social o cultural más bajo. Está implícito en el origen mismo del concepto de diglosia (variedades vulgar y culta de la misma lengua), y lo han explicitado sociólogos como Bernstein en su teoría de los códigos, o, más ampliamente, Bourdieu. Pero resulta que los datos empíricos de los que disponemos refutan de plano las conjeturas basadas en la comprensión de sentido: los alumnos no contestan los cuestionarios PISA del modo como nosotros comprenderíamos que lo hicieran, sintiéndose más encasa en los centros donde se usa su lengua doméstica, sino de un modo inesperado.

No queriendo resignarnos, proponemos la hipótesis de la diglosia escuela-sociedad. La hipótesis es que los alumnos se encuentran mejor cuando en la escuela se usa la lengua principal en el conjunto de la sociedad, no justamente en su familia. Por mucho que les agrade encontrar su lengua doméstica en la escuela cuando son niños, cuando son adolescentes les incomoda más que se use en la escuela una lengua más limitada que la del entorno social de su vida cotidiana. Dicho de otro modo, la diglosia escuela-sociedad es más importante para los adolescentes que la diglosia hogar-escuela, a diferencia de lo que seguramente ocurre con los niños.

Nótese que procuramos formular nuestra conjetura en los términos más neutros posibles, evitando incluso términos habituales en la sociolingüística, como lengua hegemónica o de prestigio. Cuando decimos que el español es la lengua 'principal' nos referimos tan solo a que es la lengua más usada incluso en aquellas zonas en que 'se vive' cotidianamente en una lengua local. La hipótesis, por tanto, se reduce a la presunción de que los adolescentes la prefieren a su lengua doméstica. La hipótesis se inspira en el bien estudiado desplazamiento de los grupos de referencia de los adolescentes desde lo familiar al grupo de iguales y en su salida de la familia a la ciudad, es decir, en su tránsito de la comunidad a la sociedad. Ahora bien, los resultados de nuestro estudio muestran bien claramente que las políticas favorecedoras de las lenguas locales no se hacen solo a costa del bienestar de los hablantes nativos de otras lenguas, sino a costa igualmente de los hablantes de la misma lengua que se quiere favorecer. Es decir, a costa del bienestar de todos los alumnos, sacrificado en aras del proyecto político de convertir las lenguas locales en lenguas hegemónicas o de prestigio.

Nuestra hipótesis es tentativa. Los datos analizados reclaman alguna, y ninguna nos parece a priori más sensata que la hasta aquí planteada, pero pasar de la asociación a la causación con certidumbre exigirá nuevas investigaciones. Las bases de datos masivas sobre bienestar escolar, sentido de pertenencia, etc. son todavía muy escasas. Para España apenas contamos con los datos de algunos informes internacionales como PISA, PIRLS y HBSC (Moreno \& al, 2016), de entre los cuales sólo PISA, en algunas de sus ediciones, permite el análisis hecho aquí, pero no ir más lejos en la explicación ni establecer relaciones propiamente causales. A pesar de la omnipresencia de temas y lemas como bienestar, pertenencia, compromiso, aburrimiento, rechazo, no nos consta la existencia de investigaciones sistemáticas en este ámbito, menos aún de otras bases de datos con cierta continuidad, salvo la excepción lejana y a día de hoy interrumpida de la High School Survey of Student Engagement realizada desde la Universidad de Indiana (Yazzie-Mintz, 2007, 2010) Nos parece altamente recomendable que los informes diagnósticos sobre el sistema educativo empiecen a incorporar datos relativos al nivel de bienestar, el sentido de pertenencia el grado de compromiso y otras variables relativas a la situación y actitud del alumnado, deseamos que pequeños o no tan pequeños estudios cualitativos nos permitan ahondar en su comprensión y confiamos en que 
nuevos análisis de los datos existentes y los que vengan nos ayuden a avanzar en la explicación de un fenómeno que a nosotros mismos nos sorprende. 


\section{BIBLIOGRAFÍA}

Allen, K. \& Bowles, T. (2013). Belonging as a guiding principle in the education of adolescents. Australian Journal of Educational \& Developmental Psychology, 12, 108-119

Allen, K., Kern, M.L., Vella-Brodrick, D., Hattie, J., Waters, L., (2018) What Schools Need to Know About Fostering School Belonging: a Meta-analysis Educational. Psychological Review, 30(1),1-34.

Álvarez Cáccamo, C. (1991). Language revival, code manipulation and social power in Galiza: Offrecord uses of Spanish in formal communicative events. En Klee, C.A. \& Ramos-García, L.(Eds.), Sociolinguistics of the Spanish-speaking world: Iberia, Latin America, United States, pp. 4173. Tempe, AZ: Bilingual Press.

Aracil, L.V.(1965)[1982]. Conflicte lingüístic i normalització lingüística a l'Europa nova. Papers de sociolingüística, pp. 23-38. Barcelona: La Magrana.

Baumeister, R.F. and M. R. Leary (1995) The Need to Belong: Desire for Interpersonal Attachments as a Fundamental Human Motivation. Psychological Bulletin, 117(3),497-529.

Bernstein, Basil (1971) Class, Codes and Control, I. .London:Basil Blackwell.

Blas Arroyo, J.L.(1999). La diglosia y otros conceptos alternativos en la descripción del bilingüismo social en comunidades hispánicas. Un ensayo bibliográfico. Grazer Linguistische Studien, 52, $19-51$

Bretxa, V. (2016) Trajectòries sociolingüístiques en la transició educativa. Revista de Llengua i Dret, Journal of Language and Law, 65:110-125.

Bretxa, V., Vila, F. X. (2013) Els Canvis sociolingüístics en el pas de primària a secundària: el projecte RESOL a la ciutat de Mataró. Treballs de Sociolingüística Catalana, 22: 93-118. https://www.raco.cat/index.php/TSC/article/view/79231 [Consulta: 22-11-2019].

Bryk, A. S., Lee, V.E. and Holland,P. B. (1993) Catholic Schools and the Common Good. Cambridge (Mass):Harvard U. P.

Calero, J, y Á. Choi. 2019. Efectos de la inmersión lingüistica sobre el alumnado castellanoparlante en Cataluña. Madrid: Fundación Europea Sociedad y Educación.

Chiu, M. M., Pong, S. L., Mori, I., \& Chow, B. W.-Y. (2012). Immigrant students' cognitive and emotional engagement at school: A multilevel analysis of students in 41 countries. Journal of Youth and Adolescence, 41, 1409-1425.

Chiu, M.M, Wing-Yin Chow, B, McBride, C. and Mol, S. T.(2016). Students' sense of belonging at school in 41 countries: Cross-cultural variability. Journal of Cross-Cultural Psychology 47(2), 175-96.

Coleman, J S, y Hoffer, T. (1987). Public and private high schools: The impact of communities. New York: Basic Books.

Coleman, J.S, Hoffer, T and Kilgore, S. (1982). High school achievement: Public, Catholic, and private schools compared. New York: Basic Books.

Cummins, J. (2009). Bilingual and Immersion Programs. Pp. 161-182 en Long, M.H and Doughty, C. J. (2009). The handbook of language teaching. Chichester: Wiley-Blackwell.

Damasio, A R. (2006). Descartes' error. New York: Random House.

Dweck, Carol S. (2008). Mindset: The new psychology of success. Random House Digital, Inc.

Feito, R. (1990).Nacidos para perder: un análisis sociológico del rechazo y del abandono escolares. Madrid: Ministerio de Educación.

Fellman, J. (1975). On diglossia. Language Sciences 34, 38-39.

Ferguson, C. A. (1959). Diglossia. Word, 15(2), 325-340.

Fernández Enguita, M. 1999. Alumnos gitanos en la escuela paya: un estudio sobre las relaciones étnicas en el sistema educativo. Barcelona: Ariel.

Fernández Enguita, M. 2011. Building the nation at school: Spain tables turned. C. Kassimeris \& M. Vryonides, The politics of education: Challenging multiculturalism. NY: Routledge. 
Fernández Enguita, M.(2018). 'Y si no te gusta, te aguantas'. En torno algunos indicadores del malestar del alumnado. Pp. 150-153 en Indicadores comentaros sobre el estado del sistema educativo español 2018. Madrid, Fundación Europea Sociedad y Educación.

Fernández Enguita, M., L. Mena Martínez, y J. Riviere Gómez. (2010). Fracaso y abandono escolar en España. Obra Social Fundación la Caixa.

Fishman, J. A. (1967). Bilingualism with and without diglossia; diglossia with and without bilingualism. Journal of Social Issues, 23(2), 29-38.

Galindo, S. M. (2008). Les Llengües en joc, el joc entre llengües: L'ús interpersonal del català entre els infants $i$ joves de Catalunya. Lleida: Pagès.

Genesee, F, Lambert, W.E., y Holobow, N. E, (1986). La adquisición de una segunda lengua mediante inmersión: el enfoque canadiense. Infancia y Aprendizaje 9(33),27-36.

Goodenow, C. (1993). Classroom belonging among early adolescent students: Relationships to motivation and achievement. The Journal of early adolescence 13(1), 21-43.

Goodenow, C, y K E Grady. (1993). The relationship of school belonging and friends' values to academic motivation among urban adolescent students. The Journal of Experimental Education 62(1), 60-71.

Gumperz, J. J. (1964). Linguistic and social interaction in two communities. American anthropologist 66(6), 137-53.

Habermas, J. (1993) La lógica de las ciencias sociales. México. Iberoamericana.

Harris, J. R (1999) El mito de la Educación. Barcelona, Grijalbo.

Henry, J. 1965. Culture against man. New York: Ramdom House.

Henry, J. 1972. Jules Henry on education. New York: Random House.

Hoffer, T, Greeley, A. M, y Coleman, J.S,(1985). Achievement growth in public and Catholic schools. Sociology of Education, 58(2), 74-97.

Hudson, A. (2002). Outline of a theory of diglossia. International Journal of the Sociology of Language, 157, 1-48.

Jackson, P W. 1968. Life in classrooms. Nueva York: Holt,. Rinehart \& Winston.

Jencks, C. 1972. Inequality: A reassessment of the effect of family and schooling in America. N. York: Harper.

Jenkins, R. 1983. Lads, citizens, and ordinary kids: Working-class youth life-styles in Belfast. Londres: Routledge.

Juvonen, J. (2006) Sense of belonging, social bonds, and school functioning, in P.A. Alexander and P.H. Winne (eds.), Handbook of Educational Psychology, Vol. 2, pp. 655-674. Mahwah, NJ, US: Lawrence Erlbaum Associates Publishers.

Mackridge, P. 2009. Language and national identity in Greece, 1766-1976. Oxford University Press.

Maslow, AH. 1943. A theory of human motivation. Psychological Review 50(4): 370.

Mega, C, L Ronconi, y R de Beni. 2014. What makes a good student? How emotions, self-regulated learning, and motivation contribute to academic achievement. Journal of educational psychology 106(1): 121.

Moreno, C., Ramos, P., García-Moya, I., Moreno-Maldonado, C., Rivera, F., Jiménez-Iglesias, A., Sánchez-Queija, I., Paniagua, C., Villafuerte-Díaz, A. y Morgan, A. (2016). Informe comparativo de las ediciones 2002-2006-2010-2014 del Estudio HBSC en España. Madrid:Ministerio de Sanidad, Servicios Sociales e Igualdad

Ninyoles, R,L(1971). Idioma y conflicto. Revista española de la opinión pública (26), 159-82.

Noddings, N. (1992). The Challenge to Care in Schools. New York: Teachers College Press.

Noddings, N. (2003). Happiness and education. Cambridge University Press.

OECD (2003), Literacy Skills for the World of Tomorrow - Further Results from PISA 2000, OECD Publishing, París.

OCDE (2005) Informe PIS A 2003. Aprender para el mundo del mañana. Madrid: Santillana.

OECD (2013). PIS A 2012 Results: Ready to Learn - Students' Engagement, Drive and Self-Beliefs (Volume III), OECD Publishing, París. 
OECD. 2017. PISA 2015 Results (Volume III): Students' Well-Being. OECD Publishing.

Osterman, K.F. (2000). Students' need for belonging in the school community. Review of Educational Research, 70(3), 323-367.

Pauwels, A. 1986. Diglossia, immigrant dialects and language maintenance in Australia: the case of Limburgs and Swabian. Journal of Multilingual \& Multicultural Development 7(1), 13-30.

Pekrun, R, T Goetz, W Titz, y R P Perry. 2002. Academic emotions in students' self-regulated learning and achievement: A program of qualitative and quantitative research. Educational psychologist 37(2), 91-105.

Plataforma per la Llengua (2019). Estudi sociolingüístic als patis d'escoles i instituts de zones urbanes de Catalunya. www.plataforma-llengua.cat.

Psichari, J \& Pernot, H (1902). The language question in Greece, Calcuta, The Baptist Mission Press.

Psycháris, G [Psichari, Jean](1929). Un pays qui ne veut pas de sa langue. Imprimerie La Haute-Loire.

Putnam, R D. (1995). Bowling alone: America's declining social capital. Journal of Democracy 6(1), 65-78.

Rhoidis, E. (1893). Ta eidola (Los ídolos). Atenas: Edissos Estia.

Rhoidis, E. (1885). Parerga (Apéndices). Atenas: Anareoy Koromhaa.

Salvador, G. (1987). Lengua española y lenguas de España. Barcelona: Ariel.

Siguan, M (ed). (1976). Bilingüisme i educación. Barcelona, Teide.

Slaten, C. D., Ferguson, J. K., Allen, K. A., Brodrick, D. V., \& Waters, L. (2016). School belonging: A review of the history, current trends, and future directions. The Educational and Developmental Psychologist, 33(1), 1-15.

Swain, M.(2000). French immersion research in Canada: Recent contributions to SLA and applied linguistics. Annual Review of Applied Linguistics 20, 199-212.

Tollefson, J. W. (1983) Language policy and the meanings of diglossia. Word, 34 (1), 1-9.

Uranga, B., ed. (2013). Comportamiento lingüsitico del alumnado. Proyecto Arrue 2011. Vitoria, Gobierno Vasco

Wentzel, K R. (1997). Student motivation in middle school: The role of perceived pedagogical caring. Journal of educational psychology 89(3), 411.

Vila, F. X. (2004) 'Hem guanyat l'escola però hem perdut el pati?'. Els usos lingüístics a les escoles catalanes». LSC- Llengua, Societat I Comunicació, 1: 8-15, https://www.raco.cat/index.php/LSC/article/view/40442 [Consulta: 22-11-2019].

Willis, P. (1978). Learning to labour: How working class kids get working class jobs. Routledge.

Willms, J.D. (2003). Student engagement at school: A sense of belonging and participation: Results from PIS A 2000. OECD publishing.

Woolard, K.A (2016) Singular and Plural. Ideologies of Linguistic Authority in 21st century Catalonia. Oxford: University Press.

Yazzie-Mintz, E. (2007). Voices of Students on Engagement: A Report on the 2006 High School Survey of Student Engagement. Center for Evaluation and Education Policy, Indiana University.

Yazzie-Mintz, E. (2010). Charting the path from engagement to achievement: A report on the 2009 High School Survey of Student Engagement. Bloomington, IN: Center for Evaluation \& Education Policy. 


\section{SOBRE LOS AUTORES}

\section{Julio Carabaña Morales}

Julio Carabaña Morales ha enseñado Sociología en la UCM desde 1976. Catedrático de Universidad desde 1990, se jubiló en 2018, siendo ahora profesor honorífico. Es autor de Educación, ocupación e ingresos en la España del Siglo XX (Madrid, MEC, 1983), Escalas de prestigio profesional (Madrid, CIS, 1996, con C. Gómez Bueno, Dos estudios sobre movilidad intergeneracional (Madrid, Argentaria-Visor, 1999), La inutilidad de PIS A para las escuelas (Catarata, 2015), Ricos y pobres (Catarata, 2016) y de varios artículos y capítulos de libros sobre Sociología de la Educción, Estratificación y movilidad social y desigualdad de ingresos.

Información de contacto: Universidad Complutense de Madrid, Facultad de Educación, Departamento de Sociología Aplicada, carabanya@ccedu.ucm.es

\section{Mariano Fernández Enguita}

Catedrático de Sociología en la Universidad Complutense, donde coordina el Doctorado en Educación y diseñó y anima el proyecto de innovación hiperaula.ucm. Antes lo fue en Salamanca, donde dirigió el Departamento de Sociología , el Centro de Análisis Sociales (investigación) y el Centro Cultural Hispano Japonés (la Casa de Japón en España) y creó los portales Demos (docencia) e Innova (innovación). Autor de una veintena de libros y tres centenares de artículos y capítulos. Últimos libros: La educación en la encrucijada (2016, Santillana) Del clip al clic (2017, Ariel, con S. Vázquez), Más escuela y menos aula. La innovación educativa en un cambio de época (2018, Morata). Actualmente investiga sobre la profesión docente y sobre aprendizaje y escuela en la era informacional, participa en la Red por el Diálogo Educativo (REDE) e impulsa el proyecto hiperaula.ucm. Blog: Cuaderno de Campo. Más: @enguita,www.enguita.info

Información de contacto: Universidad Complutense de Madrid, Facultad de Educación, Departamento de Sociología Aplicada,mfenguita@ucm.es 


\section{ANEXOS}

Tabla 1. Lengua escolar, lengua doméstica, sentimiento de pertenencia a la escuela y puntuación PISA

Ciencias. Países participantes en PISA 2015 con más de un 3\% de diglosia hogar-escuela.

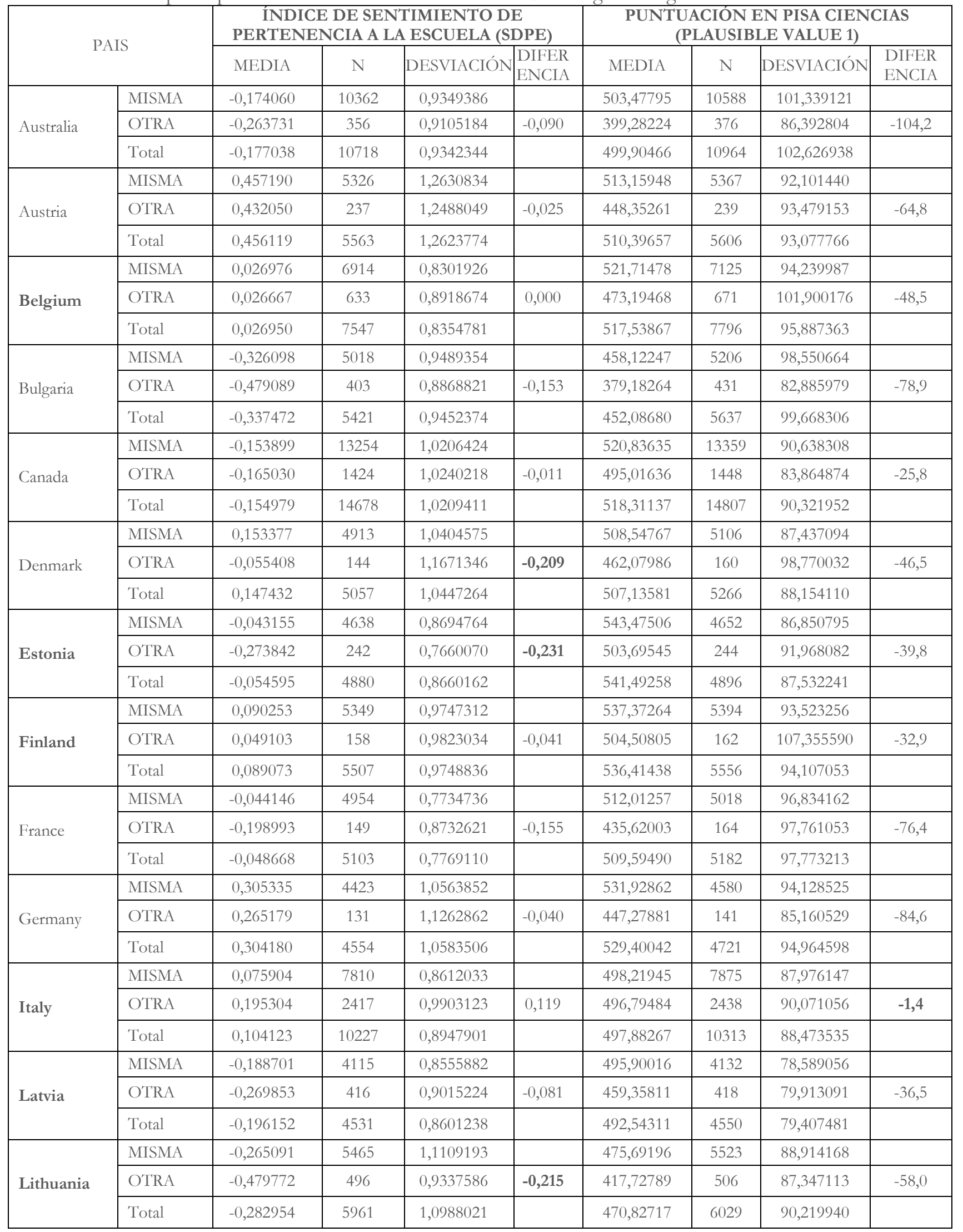


Journal of Supranational POlicies of EduCAtion no 9 , pp. 57 - 94

\begin{tabular}{|c|c|c|c|c|c|c|c|c|c|}
\hline \multirow{3}{*}{$\begin{array}{l}\text { Luxem } \\
\text { bourg }\end{array}$} & MISMA & 0,072763 & 160 & 0,9727728 & & 513,91985 & 162 & 99,258756 & \\
\hline & OTRA & 0,308117 & 2313 & 1,1115221 & 0,235 & 505,31486 & 2334 & 90,642437 & $-8,6$ \\
\hline & Total & 0,292890 & 2473 & 1,1044156 & & 505,87336 & 2496 & 91,229599 & \\
\hline \multirow{3}{*}{ Macao } & MISMA & $-0,395836$ & 1269 & 0,6305485 & & 523,40429 & 1269 & 81,262289 & \\
\hline & OTRA & $-0,502699$ & 399 & 0,4943307 & $-0,107$ & 503,73617 & 400 & 88,499386 & $-19,7$ \\
\hline & Total & $-0,421399$ & 1668 & 0,6023692 & & 518,69054 & 1669 & 83,450712 & \\
\hline \multirow{3}{*}{ Mexico } & MISMA & $-0,124455$ & 7059 & 1,0940507 & & 424,69743 & 7095 & 69,720676 & \\
\hline & OTRA & $-0,371937$ & 212 & 0,9840638 & $-0,247$ & 356,65044 & 219 & 66,429357 & $-68,0$ \\
\hline & Total & $-0,131670$ & 7271 & 1,0917337 & & 422,65993 & 7314 & 70,579478 & \\
\hline \multirow{3}{*}{ Peru } & MISMA & $-0,180380$ & 6210 & 0,8097071 & & 403,89911 & 6326 & 74,115303 & \\
\hline & OTRA & $-0,579394$ & 463 & 0,6504984 & $-0,399$ & 334,92662 & 494 & 63,752263 & $-69,0$ \\
\hline & Total & $-0,208065$ & 6673 & 0,8060468 & & 398,90316 & 6820 & 75,555604 & \\
\hline \multirow{3}{*}{ Qatar } & MISMA & $-0,121462$ & 3731 & 1,0475467 & & 372,48904 & 3996 & 75,681269 & \\
\hline & OTRA & $-0,180326$ & 1075 & 0,9248252 & $-0,059$ & 388,85462 & 1156 & 91,829569 & 16,4 \\
\hline & Total & $-0,134629$ & 4806 & 1,0215795 & & 376,16113 & 5152 & 79,873051 & \\
\hline \multirow{3}{*}{$\begin{array}{l}\text { Russian } \\
\text { Federation }\end{array}$} & MISMA & $-0,365370$ & 5011 & 0,7943270 & & 490,09624 & 5104 & 83,716177 & \\
\hline & OTRA & $-0,477999$ & 268 & 0,6834519 & $-0,113$ & 451,72060 & 280 & 78,088501 & $-38,4$ \\
\hline & Total & $-0,371087$ & 5279 & 0,7894040 & & 488,10048 & 5384 & 83,860121 & \\
\hline \multirow{3}{*}{$\begin{array}{l}\text { Singa } \\
\text { pore }\end{array}$} & MISMA & $-0,189402$ & 2606 & 0,9094308 & & 567,82923 & 2608 & 97,643895 & \\
\hline & OTRA & $-0,283198$ & 2210 & 0,8160138 & $-0,094$ & 509,25263 & 2217 & 103,393296 & $-58,6$ \\
\hline & Total & $-0,232444$ & 4816 & 0,8689820 & & 540,91435 & 4825 & 104,478043 & \\
\hline \multirow{3}{*}{ Spain } & MISMA & 0,566038 & 4934 & 1,1501940 & & 503,32959 & 5059 & 84,420695 & \\
\hline & OTRA & 0,209214 & 815 & 1,1477330 & $-0,357$ & 500,93037 & 826 & 85,335598 & $-2,4$ \\
\hline & Total & 0,515453 & 5749 & 1,1564640 & & 502,99284 & 5885 & 84,546516 & \\
\hline \multirow{3}{*}{ Sweden } & MISMA & 0,072864 & 4149 & 1,2135918 & & 508,34346 & 4192 & 95,979735 & \\
\hline & OTRA & $-0,085680$ & 179 & 1,2130500 & $-0,159$ & 483,23219 & 190 & 120,805758 & $-25,1$ \\
\hline & Total & 0,066307 & 4328 & 1,2138399 & & 507,25465 & 4382 & 97,305442 & \\
\hline \multirow{3}{*}{$\begin{array}{l}\text { Switzer } \\
\text { land }\end{array}$} & MISMA & 0,393609 & 3626 & 1,0391042 & & 525,32498 & 3646 & 90,408418 & \\
\hline & OTRA & 0,271419 & 300 & 1,2176444 & $-0,122$ & 477,80445 & 304 & 96,099282 & $-47,5$ \\
\hline & Total & 0,384272 & 3926 & 1,0541396 & & 521,66771 & 3950 & 91,725195 & \\
\hline \multirow{3}{*}{$\begin{array}{l}\text { United } \\
\text { Arab } \\
\text { Emirates }\end{array}$} & MISMA & $-0,081688$ & 4436 & 0,8451596 & & 389,05382 & 4561 & 79,613557 & \\
\hline & OTRA & $-0,180167$ & 1367 & 0,8889950 & $-0,098$ & 414,62685 & 1410 & 86,958860 & 25,6 \\
\hline & Total & $-0,104886$ & 5803 & 0,8566303 & & 395,09267 & 5971 & 82,121914 & \\
\hline \multirow{3}{*}{ Tunisia } & MISMA & $-0,176561$ & 4450 & 0,7352601 & & 388,62516 & 4590 & 64,762527 & \\
\hline & OTRA & $-0,309085$ & 275 & 0,7775964 & $-0,133$ & 384,79700 & 295 & 62,382822 & $-3,8$ \\
\hline & Total & $-0,184274$ & 4725 & 0,7383568 & & 388,39398 & 4885 & 64,621546 & \\
\hline \multirow{3}{*}{ Turkey } & MISMA & $-0,421624$ & 5256 & 1,1193905 & & 427,18760 & 5298 & 76,505761 & \\
\hline & OTRA & $-0,561930$ & 392 & 1,0995266 & $-0,140$ & 380,52530 & 398 & 67,251250 & $-46,7$ \\
\hline & Total & $-0,431362$ & 5648 & 1,1184959 & & 423,92714 & 5696 & 76,817289 & \\
\hline \multirow{3}{*}{$\begin{array}{l}\text { United } \\
\text { Kingdom }\end{array}$} & MISMA & $-0,074352$ & 11128 & 0,9063664 & & 509,04256 & 11274 & 95,779376 & \\
\hline & OTRA & $-0,155886$ & 384 & 0,8010191 & $-0,082$ & 466,22237 & 388 & 89,763948 & $-42,8$ \\
\hline & Total & $-0,077072$ & 11512 & 0,9031381 & & 507,61791 & 11662 & 95,889728 & \\
\hline \multirow{2}{*}{$\begin{array}{l}\text { United } \\
\text { States }\end{array}$} & MISMA & $-0,040669$ & 3987 & 1,0511954 & & 506,99369 & 4046 & 96,597309 & \\
\hline & OTRA & $-0,119636$ & 148 & 1,0100126 & $-0,079$ & 448,68236 & 152 & 90,596760 & $-58,3$ \\
\hline
\end{tabular}




\begin{tabular}{|c|c|c|c|c|c|c|c|c|c|}
\hline & Total & $-0,043495$ & 4135 & 1,0497339 & & 504,88237 & 4198 & 96,990146 & \\
\hline \multirow{3}{*}{ Uruguay } & MISMA & $-0,060569$ & 5292 & 1,1053807 & & 440,19707 & 5495 & 87,811358 & \\
\hline & OTRA & $-0,261906$ & 206 & 1,0752744 & $-0,201$ & 394,79172 & 219 & 75,728357 & $-45,4$ \\
\hline & Total & $-0,068112$ & 5498 & 1,1048340 & & 438,45683 & 5714 & 87,807079 & \\
\hline \multirow{3}{*}{$\begin{array}{l}\text { Spain } \\
\text { (Regions) }\end{array}$} & MISMA & 0,580536 & 24306 & 1,1347167 & & 504,77411 & 24385 & 85,146027 & \\
\hline & OTRA & 0,237671 & 3755 & 1,0888559 & $-0,343$ & 501,61449 & 3774 & 85,194415 & $-3,2$ \\
\hline & Total & 0,534656 & 28061 & 1,1346893 & & 504,35064 & 28159 & 85,157804 & \\
\hline \multirow{3}{*}{ Total } & MISMA & 0,035354 & 180151 & 1,0448666 & & 488,64803 & 188021 & 98,971050 & \\
\hline & OTRA & $-0,030916$ & 21967 & 1,0242486 & $-0,066$ & 468,91009 & 22734 & 101,370067 & $-19,7$ \\
\hline & Total & 0,028151 & 202118 & 1,0428470 & & 486,51891 & 210755 & 99,421105 & \\
\hline
\end{tabular}

Fuente: Datos PISA, 2015 
Tabla 2a. Relación entre SdPE, inmigración y diglosia. España, 2015

\begin{tabular}{|l|l|r|r|c|}
\hline \multicolumn{2}{|l|}{ INMIGRANTE } & \multicolumn{1}{c|}{ MEDIA } & N & $\begin{array}{c}\text { DESV. } \\
\text { TÍP. }\end{array}$ \\
\hline \multirow{4}{*}{ NO } & MONOGLOSICO &, 552903 & 27954 & 1,1474709 \\
\cline { 2 - 5 } & DIGLOSICO &, 271625 & 5310 & 1,1324754 \\
\cline { 2 - 5 } & Total &, 508000 & 33264 & 1,1496987 \\
\hline \multirow{4}{*}{$S i ́$} & MONOGLOSICO &, 330276 & 2011 & 1,0587306 \\
\cline { 2 - 5 } & DIGLOSICO &, 038502 & 2284 & 1,0960821 \\
\cline { 2 - 5 } Total & Total &, 175108 & 4295 & 1,0884140 \\
\hline \multirow{3}{*}{} & MONOGLOSICO &, 537964 & 29965 & 1,1430735 \\
\cline { 2 - 5 } & DIGLOSICO &, 201515 & 7594 & 1,1266667 \\
\cline { 2 - 5 } & Total &, 469936 & 37559 & 1,1477431 \\
\hline
\end{tabular}

*Ponderación nacional, todos

Tabla 2b. Relación entre SdPE, lengua extranjera y diglosia. España, 2015

\begin{tabular}{|l|l|r|r|r|}
\hline \multicolumn{2}{|l|}{ LENGUA DOMÉSTICA } & \multicolumn{1}{c|}{ MEDIA } & N & \multicolumn{1}{c|}{$\begin{array}{c}\text { DESV. } \\
\text { TÍP. }\end{array}$} \\
\hline \multirow{3}{*}{$\begin{array}{l}\text { LENGUA } \\
\text { ESPANOLA }\end{array}$} & MONOGLOSICO &, 552903 & 27954 & 1,1474709 \\
\cline { 2 - 5 } & DIGLOSICO &, 284126 & 5007 & 1,1252975 \\
\cline { 2 - 5 } & Total &, 512072 & 32961 & 1,1481736 \\
\hline \multirow{2}{*}{$\begin{array}{l}\text { EXNGUA ANJERA } \\
\text { Total }\end{array}$} & DIGLOSICO &, 065089 & 303 & 1,2287967 \\
\cline { 2 - 5 } & Total &, 065089 & 303 & 1,2287967 \\
\hline \multirow{2}{*}{ MONOGLOSICO } &, 271625 & 5310 & 1,1324754 \\
\cline { 2 - 5 } & DIGLOSICO &, 508000 & 33264 & 1,1496987 \\
\cline { 2 - 5 } & Total & & & \\
\hline
\end{tabular}

*Ponderación nacional, solo nativos

Fuente: Datos PISA, 2015 
Tabla 3A. Sentido de pertenencia a la escuela (SdPE) por

CCAA, España, 2003.

SENTIDO DE PERTENENCIA A LA ESCUELA (SdPE)

\begin{tabular}{|l|r|r|r|}
\hline REGION & \multicolumn{1}{|c|}{ Media } & \multicolumn{1}{c|}{ N } & Desviación típica \\
\hline RESTO & 0,277070 & 2541 & 1,0116405 \\
\hline CASTILLA-LEÓN & 0,323413 & 1484 & 0,9677913 \\
\hline CATALUÑA & $-\mathbf{0 , 0 8 9 8 0 0}$ & 1508 & 0,9267963 \\
\hline CA VASCA & 0,106227 & 3870 & 0,9334280 \\
\hline ANDALUCIA & 0,274518 & 1334 & 1,0221098 \\
\hline Total & 0,170054 & 10737 & 0,9767551 \\
\hline
\end{tabular}

Nota:Ponderación nacional, solo nativos de lengua doméstica española.

Tabla 3B. Sentido de pertenencia a la escuela(SdPE) por CCAA, España, 2012.

SENTIDO DE PERTENENCIA A LA ESCUELA (SdPE)

\begin{tabular}{|c|c|c|c|}
\hline CCAA & Media & $\mathbf{N}$ & Desv. típ. \\
\hline ANDALUCIA &, 5836 & 947 & 1,07589 \\
\hline ARAGON & ,4326 & 898 & 1,11476 \\
\hline ASTURIAS & ,4752 & 1074 & 1,14807 \\
\hline BALEARES & ,2211 & 909 & 1,07002 \\
\hline CANTABRIA & ,4884 & 1000 & 1,09380 \\
\hline CASTILLA-L & ,4371 & 1034 & 1,04544 \\
\hline CATALUÑA & 1253 & 927 & ,99592 \\
\hline EXTREMADURA &, 5369 & 1007 & 1,09549 \\
\hline GALICIA & ,0133 & 1011 & ,97928 \\
\hline RIOJA & ,4360 & 1009 & 1,06866 \\
\hline MADRID &, 5297 & 1020 & 1,08223 \\
\hline MURCIA & ,4481 & 888 & 1,08940 \\
\hline NAVARRA & ,2686 & 1012 & 1,00229 \\
\hline CA VASCA & 1135 & 3085 & 95846 \\
\hline RESTO &, 5017 & 702 & 1,10013 \\
\hline Total &, 3399 & 16524 & 1,06481 \\
\hline \multicolumn{3}{|c|}{ Media CCAA monolingües } & 0,4869 \\
\hline \multicolumn{3}{|c|}{ Media CCAA bilingües } & 0,1483 \\
\hline \multicolumn{3}{|l|}{ Diferencia } & 0,3386 \\
\hline
\end{tabular}

Nota:Ponderación nacional, solo nativos de lengua doméstica española. 
Tabla 3C. Sentido de pertenencia a la escuela(SdPE) por CCAA, España, 2015.

SENTIDO DE PERTENENCIA A LA ESCUELA (SdPE)

\begin{tabular}{|c|c|c|c|}
\hline CCAA & Media & $\mathbf{N}$ & Desv. típ. \\
\hline ANDALUCIA &, 594961 & 7357 & 1,1155899 \\
\hline ARAGON & ,654845 & 901 & 1,1244559 \\
\hline ASTURIAS & 641570 & 601 & 1,1987691 \\
\hline BALEARES & ,202276 & 686 & 1,1337705 \\
\hline CANARIAS &, 502409 & 1595 & 1,1820515 \\
\hline CANTABRIA & ,751996 & 324 & 1,1294756 \\
\hline CASILLA-L & ,771902 & 1170 & 1,1643439 \\
\hline CASTILLA-M & ,601039 & 1688 & 1,1256203 \\
\hline CATALUÑA & ,230478 & 4898 & 1,1644533 \\
\hline EXTREMADURA &, 596794 & 966 & 1,1277885 \\
\hline GALICIA & ,251247 & 1668 & 1,1048983 \\
\hline RIOJA &, 545588 & 219 & 1,1068553 \\
\hline MADRID &, 574311 & 4198 & 1,1511044 \\
\hline MURCIA &, 597743 & 1068 & 1,0808622 \\
\hline NAVARRA &, 568865 & 483 & 1,0717479 \\
\hline CA VASCA & ,496466 & 1467 & 1,1104229 \\
\hline C. VALENCIANA &, 537363 & 3076 & 1,1567108 \\
\hline Total &, 506835 & 32366 & 1,1478589 \\
\hline
\end{tabular}

Nota:Ponderación nacional, solo nativos de lengua doméstica española.

Fuente: Datos PISA, 2015 
Tabla 4. Sentido de pertenencia a la escuela(SdPE) y diglosia hogar-escuela. España, 2015.

\begin{tabular}{|c|c|c|c|c|c|}
\hline \multicolumn{2}{|l|}{ CCAA/DIGLOSI2 } & Media & $\mathbf{N}$ & Desv. típ. & Diferencia \\
\hline \multirow{3}{*}{ BALEARES } & MONOGLOSICOS & ,206176 & 386 & 1,1063460 & \\
\hline & DIGLOSICOS & ,197248 & 300 & 1,1700210 & $-0,008929$ \\
\hline & Total & ,202276 & 686 & 1,1337705 & \\
\hline \multirow{3}{*}{ CATALUÑA } & MONOGLOSICOS &, 259964 & 2427 & 1,1870627 & \\
\hline & DIGLOSICOS &, 201525 & 2471 & 1,1413161 & $-0,058439$ \\
\hline & Total &, 230478 & 4898 & 1,1644533 & \\
\hline \multirow{3}{*}{ GALICIA } & MONOGLOSICOS & ,326492 & 1009 & 1,1821517 & \\
\hline & DIGLOSICOS &, 135905 & 659 & ,9641902 & $-0,190588$ \\
\hline & Total &, 251247 & 1668 & 1,1048983 & \\
\hline \multirow{3}{*}{ NAVARRA } & MONOGLOSICOS &, 639245 & 343 & 1,1166744 & \\
\hline & DIGLOSICOS &, 396094 & 140 & ,9339292 & $-0,243151$ \\
\hline & Total &, 568865 & 483 & 1,0717479 & \\
\hline \multirow{3}{*}{ CA VASCA } & MONOGLOSICOS &, 455359 & 721 & 1,1194344 & \\
\hline & DIGLOSICOS &, 536164 & 746 & 1,1009439 & 0,080805 \\
\hline & Total &, 496466 & 1467 & 1,1104229 & \\
\hline \multirow{3}{*}{ C. VALENCIANA } & MONOGLOSICOS &, 558829 & 2384 & 1,1473455 & \\
\hline & DIGLOSICOS &, 463403 & 692 & 1,1862851 & $-0,095426$ \\
\hline & Total &, 537363 & 3076 & 1,1567108 & \\
\hline \multirow{3}{*}{ Total CCAA bilingües } & MONOGLOSICOS &, 401606 & 7270 & 1,1678291 & \\
\hline & DIGLOSICOS &, 284126 & 5007 & 1,1252975 & $-0,117480$ \\
\hline & Total & ,353691 & 12277 & 1,1520735 & \\
\hline RESTO & MONOGLOSICOS & ,606079 & 20684 & 1,1354800 & \\
\hline
\end{tabular}

Nota: Ponderación nacional, solo nativos de lengua doméstica española.

Fuente: Datos PISA, 2015 
Tabla 5. Sentido de pertenencia a la escuela(SdPE) y diglosia. España, 2015

\begin{tabular}{|c|c|c|c|c|}
\hline \multicolumn{2}{|c|}{ ZONA /DIGLOSIA } & Media & $\mathbf{N}$ & Desv. típ. \\
\hline \multirow{2}{*}{$\begin{array}{l}\text { UNA } \\
\text { LENGUA }\end{array}$} & NO, ESPAÑOL & 606079 & 20684 & 1,1354800 \\
\hline & Total & 606079 & 20684 & 1,1354800 \\
\hline \multirow{5}{*}{$\begin{array}{l}\text { DOS } \\
\text { LENGUAS }\end{array}$} & NO, ESPAÑOL &, 588146 & 3299 & 1,1478052 \\
\hline & NO, REGIONAL & ,246591 & 3970 & 1,1617827 \\
\hline & $\begin{array}{l}\text { SI, ESPAÑOL- } \\
\text { REGIONAL }\end{array}$ & ,233040 & 4442 & 1,1248571 \\
\hline & $\begin{array}{l}\text { SI,REGIONAL } \\
\text { ESPAÑOL }\end{array}$ & ,685463 & 565 & 1,0461540 \\
\hline & Total & ,353691 & 12277 & 1,1520735 \\
\hline \multirow{5}{*}{ Total } & NO, ESPAÑOL & ,603612 & 23984 & 1,1371761 \\
\hline & NO, REGIONAL & ,246591 & 3970 & 1,1617827 \\
\hline & $\begin{array}{l}\text { SI, ESPAÑOL- } \\
\text { REGIONAL }\end{array}$ & ,233040 & 4442 & 1,1248571 \\
\hline & $\begin{array}{l}\text { SI,REGIONAL } \\
\text { ESPAÑOL }\end{array}$ & ,685463 & 565 & 1,0461540 \\
\hline & Total &, 512072 & 32961 & 1,1481736 \\
\hline
\end{tabular}

Nota: Ponderación nacional, solo nativos de lengua doméstica española.

Fuente: Datos PISA, 2015 
Tabla 6. Determinantes del sentimiento de pertenencia a la escuela (SdPE). España*, 2015

\begin{tabular}{|c|c|c|c|c|c|c|}
\hline & \multirow[t]{2}{*}{ MODELO } & \multicolumn{2}{|c|}{$\begin{array}{c}\text { COEFICIENTES } \\
\text { NO } \\
\text { ESTANDARIZADOS } \\
\end{array}$} & \multirow{2}{*}{$\begin{array}{c}\text { COEFICIENTES } \\
\text { TIPIFICADOS } \\
\text { Beta }\end{array}$} & \multirow[t]{2}{*}{$\mathbf{T}$} & \multirow[t]{2}{*}{ SIG. } \\
\hline & & $\mathrm{B}$ & Error típ. & & & \\
\hline \multirow{2}{*}{$1 \mathrm{R}^{2}=1,1 \%$} & (Constante) & ,600 & ,008 & & 74,447 & 0,000 \\
\hline & ZONA BILINGÜE* &,- 245 & 013 &,- 104 & $-18,706$ &, 000 \\
\hline \multirow{5}{*}{$2 \mathrm{R}^{2}=1,9 \%$} & (Constante) & ,600 &, 008 & & 74,762 & 0,000 \\
\hline & ZONA BILINGÜE &,- 007 & ,021 &,- 003 &,- 347 &, 728 \\
\hline & ESPAÑOL-REGIONAL &,- 360 &, 026 &,- 108 & $-13,738$ &, 000 \\
\hline & REGIONAL-REGIONAL &,- 345 &, 027 &,- 098 & $-12,846$ &, 000 \\
\hline & REGIONAL-ESPAÑOL & ,093 &, 052 & 011 & 1,789 &, 074 \\
\hline \multirow{8}{*}{$3 \mathrm{R}^{2}=2,5 \%$} & (Constante) & ,746 & ,041 & & 18,034 &, 000 \\
\hline & ZONA BILINGÜE &,- 031 &, 022 &,- 013 & $-1,451$ & 147 \\
\hline & ESPAÑOL-REGIONAL &,- 340 & ,026 &,- 102 & $-12,977$ &, 000 \\
\hline & REGIONAL-REGIONAL &,- 344 &, 027 &,- 098 & $-12,715$ &, 000 \\
\hline & REGIONAL-ESPAÑOL &, 099 &, 052 &, 011 & 1,916 &, 055 \\
\hline & CENTRO PRIVADO &, 056 &, 014 & ,023 & 3,916 &, 000 \\
\hline & ESCS &, 070 &, 006 &, 072 & 11,458 &, 000 \\
\hline & PISA CIENCIAS & ,000 & ,000 &,- 019 & $-3,248$ & ,001 \\
\hline
\end{tabular}

*CCAA bilingües agrupadas.

Nota: Ponderación nacional, solo nativos de lengua doméstica española.

Fuente: Datos PISA, 2015 
Tabla 7. Cuatro situaciones de lengua doméstica y escolar, por CCAA, 2015.

\begin{tabular}{|c|c|c|c|c|c|}
\hline \multirow[b]{2}{*}{ CCAA } & \multicolumn{4}{|c|}{ DIGLOSIA } & \multirow[b]{2}{*}{ TOTAL } \\
\hline & $\begin{array}{c}\text { NO, } \\
\text { ESPAÑ̃L }\end{array}$ & $\begin{array}{c}\text { NO, } \\
\text { REGIONAL }\end{array}$ & $\begin{array}{c}\text { SI, } \\
\text { ESPAÑOL- } \\
\text { REGIONAL }\end{array}$ & $\begin{array}{c}\text { SI, } \\
\text { REGIONAL- } \\
\text { ESPAÑOL }\end{array}$ & \\
\hline \multirow{2}{*}{ BALEARES } & 122 & 716 & 595 & 57 & 1490 \\
\hline & $8,2 \%$ & $48,1 \%$ & $39,9 \%$ & $3,8 \%$ & $100,0 \%$ \\
\hline \multirow{2}{*}{ CATALUÑA } & 0 & 1077 & 1106 & 0 & 2183 \\
\hline & $0,0 \%$ & $49,3 \%$ & $50,7 \%$ & $0,0 \%$ & $100,0 \%$ \\
\hline \multirow{2}{*}{ GALICIA } & 515 & 660 & 646 & 118 & 1939 \\
\hline & $26,6 \%$ & $34,0 \%$ & $33,3 \%$ & $6,1 \%$ & $100,0 \%$ \\
\hline \multirow{2}{*}{ NAVARRA } & 1193 & 51 & 421 & 85 & 1750 \\
\hline & $68,2 \%$ & $2,9 \%$ & $24,1 \%$ & $4,9 \%$ & $100,0 \%$ \\
\hline \multirow{2}{*}{ CA VASCA } & 1085 & 666 & 1781 & 27 & 3559 \\
\hline & $30,5 \%$ & $18,7 \%$ & $50,0 \%$ &, $8 \%$ & $100,0 \%$ \\
\hline \multirow{2}{*}{ C. VALENCIANA } & 1184 & 212 & 168 & 237 & 1801 \\
\hline & $65,7 \%$ & $11,8 \%$ & $9,3 \%$ & $13,2 \%$ & $100,0 \%$ \\
\hline \multirow{2}{*}{ Total } & 4099 & 3382 & 4717 & 524 & 12722 \\
\hline & $32,2 \%$ & $26,6 \%$ & $37,1 \%$ & $4,1 \%$ & $100,0 \%$ \\
\hline
\end{tabular}

Nota: Ponderación por CA, CCAA bilingües, solo nativos de lengua doméstica española. Fuente: Datos PISA, 2015 
Tabla 8. Determinantes del sentimiento de pertenencia a la escuela (SdPE), por CCAA, 2015

Tabla 8A. BALEARES

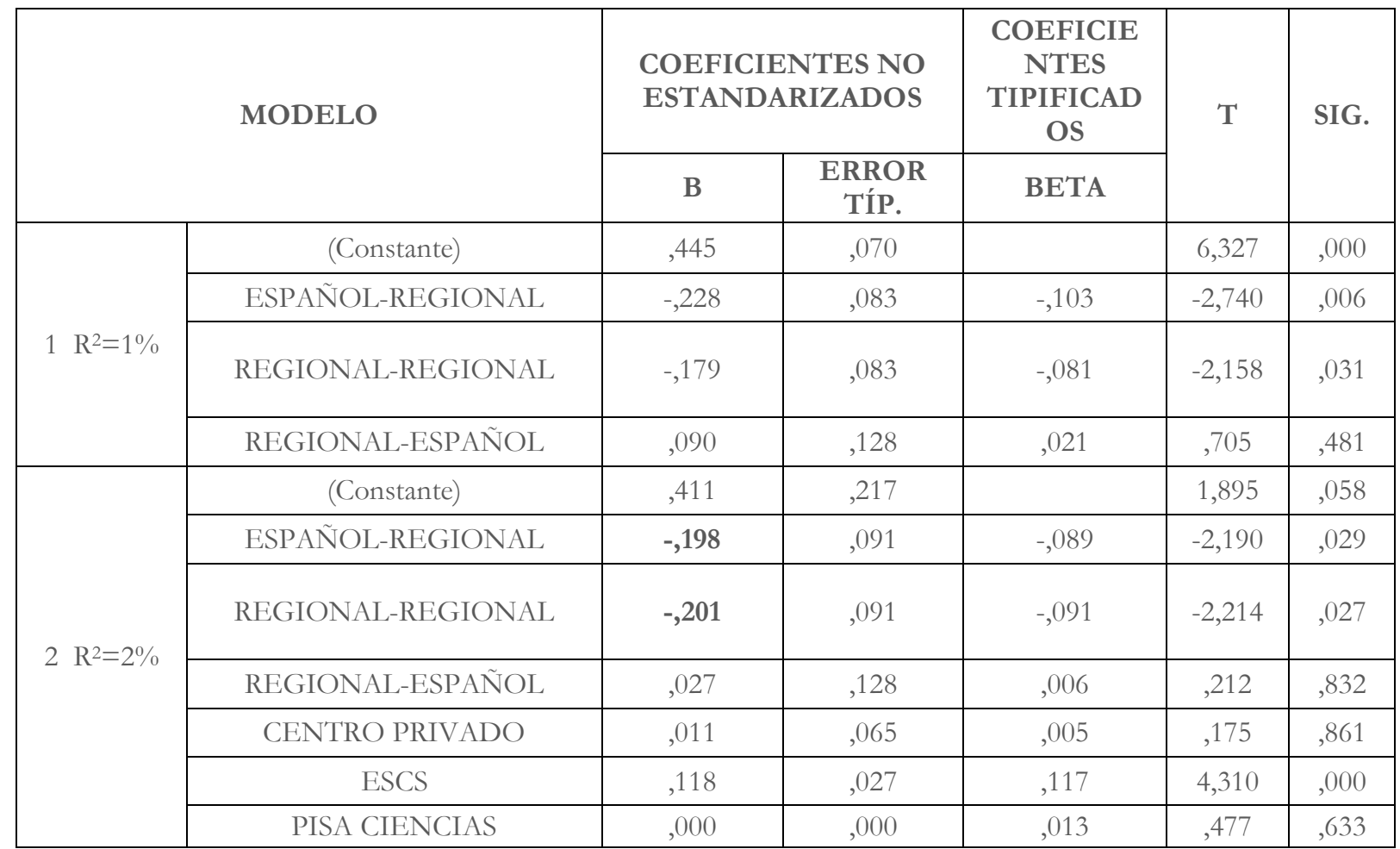

Tabla 8.b GALICIA

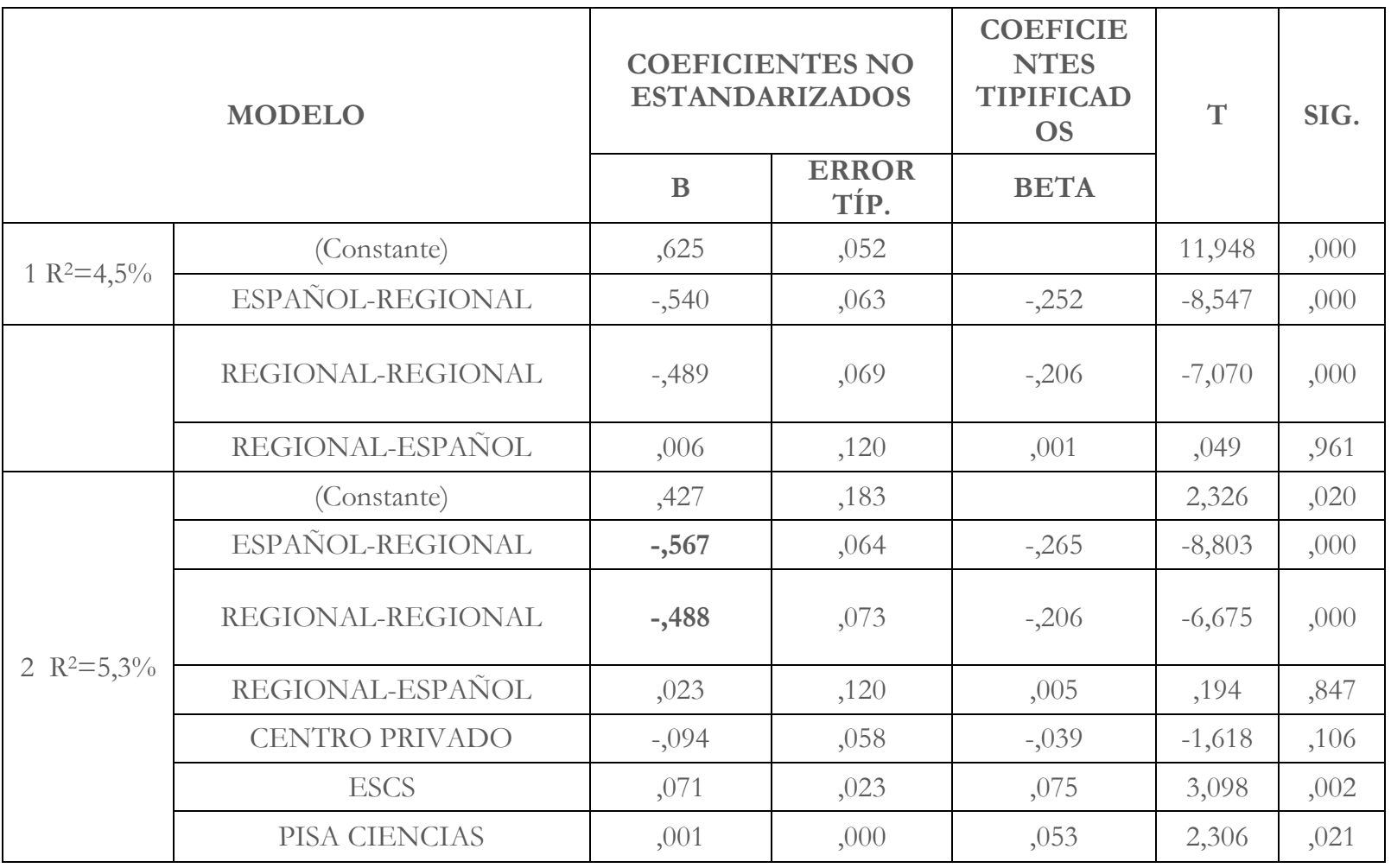


Tabla 8C.NAVARRA

\begin{tabular}{|c|c|c|c|c|c|c|}
\hline & \multirow[t]{2}{*}{ MODELO } & \multicolumn{2}{|c|}{$\begin{array}{l}\text { COEFICIENTES NO } \\
\text { ESTANDARIZADOS }\end{array}$} & \multirow{2}{*}{$\begin{array}{c}\text { COEFICIE } \\
\text { NTES } \\
\text { TIPIFICAD } \\
\text { OS } \\
\text { BETA }\end{array}$} & \multirow[t]{2}{*}{$\mathbf{T}$} & \multirow[t]{2}{*}{ SIG. } \\
\hline & & B & $\begin{array}{c}\text { ERROR } \\
\text { TÍP. }\end{array}$ & & & \\
\hline \multirow{4}{*}{$1 \mathrm{R}^{2}=2,9 \%$} & (Constante) & 661 & ,032 & & 20,916 & ,000 \\
\hline & ESPAÑOL-REGIONAL &,- 447 & ,068 &,- 158 & $-6,533$ & 000 \\
\hline & REGIONAL-REGIONAL &,- 479 & 120 &,- 096 & $-3,984$ &, 000 \\
\hline & REGIONAL-ESPAÑOL &,- 183 & ,151 &,- 029 & $-1,209$ & 227 \\
\hline \multirow{7}{*}{$2 \mathrm{R}^{2}=3,7 \%$} & (Constante) &, 330 & 201 & & 1,644 &, 100 \\
\hline & ESPAÑOL-REGIONAL &,- 432 & 069 &,- 153 & $-6,288$ & ,000 \\
\hline & REGIONAL-REGIONAL &,- 443 & ,122 &,- 089 & $-3,647$ & ,000 \\
\hline & REGIONAL-ESPAÑOL &,- 114 & , 152 &,- 018 &,- 748 & ,454 \\
\hline & CENTRO PRIVADO & ,019 & ,059 &, 009 & ,328 &, 743 \\
\hline & ESCS & ,083 & ,029 & ,076 & 2,886 &, 004 \\
\hline & PISA CIENCIAS & ,001 & 000 & 043 & 1,688 & ,092 \\
\hline
\end{tabular}

Tabla 8D. CA VASCA

\begin{tabular}{|c|c|c|c|c|c|c|}
\hline & \multirow[t]{2}{*}{ MODELO } & \multicolumn{2}{|c|}{$\begin{array}{l}\text { COEFICIENTES NO } \\
\text { ESTANDARIZADOS }\end{array}$} & \multirow{2}{*}{$\begin{array}{c}\text { COEFICIE } \\
\text { NTES } \\
\text { TIPIFICAD } \\
\text { OS } \\
\text { BETA }\end{array}$} & \multirow[t]{2}{*}{$\mathbf{T}$} & \multirow[t]{2}{*}{ SIG. } \\
\hline & & B & $\begin{array}{c}\text { ERROR } \\
\text { TÍP. }\end{array}$ & & & \\
\hline \multirow{4}{*}{$1 \mathrm{R}^{2}=0,3 \%$} & (Constante) &, 573 & 029 & & 19,914 & ,000 \\
\hline & ESPAÑOL-REGIONAL &,- 094 & ,041 &,- 042 & $-2,278$ & ,023 \\
\hline & REGIONAL-REGIONAL &,- 163 & 054 &,- 055 & $-3,010$ &, 003 \\
\hline & REGIONAL-ESPAÑOL & ,346 & 199 & ,030 & 1,738 & ,082 \\
\hline \multirow{7}{*}{$2 \mathrm{R}^{2}=1,1 \%$} & (Constante) & ,340 & 145 & & 2,342 & ,019 \\
\hline & ESPAÑOL-REGIONAL &,- 057 & ,046 &,- 025 & $-1,217$ & ,224 \\
\hline & REGIONAL-REGIONAL &,- 157 & ,057 &,- 053 & $-2,733$ &, 006 \\
\hline & REGIONAL-ESPAÑOL & ,349 & , 198 &, 030 & 1,763 &, 078 \\
\hline & CENTRO PRIVADO & ,004 & ,045 &, 002 &, 090 & ,929 \\
\hline & ESCS & ,082 & ,019 & ,079 & 4,335 &, 000 \\
\hline & PISA CIENCIAS & 000 & ,000 & 033 & 1,853 &, 064 \\
\hline
\end{tabular}


Tabla 8E. COMUNIDAD VALENCIANA

\begin{tabular}{|c|c|c|c|c|c|c|}
\hline & \multirow[t]{2}{*}{ MODELO } & \multicolumn{2}{|c|}{$\begin{array}{l}\text { COEFICIENTES NO } \\
\text { ESTANDARIZADOS }\end{array}$} & \multirow{2}{*}{$\begin{array}{c}\text { COEFICIE } \\
\text { NTES } \\
\text { TIPIFICAD } \\
\text { OS } \\
\text { BETA }\end{array}$} & \multirow[t]{2}{*}{$\mathbf{T}$} & \multirow[t]{2}{*}{ SIG. } \\
\hline & & B & $\begin{array}{c}\text { ERROR } \\
\text { TÍP. }\end{array}$ & & & \\
\hline \multirow{4}{*}{$1 \mathrm{R}^{2}=1,5 \%$} & (Constante) &, 575 & ,033 & & 17,234 &, 000 \\
\hline & ESPAÑOL-REGIONAL &,- 429 & ,097 &,- 105 & $-4,418$ &, 000 \\
\hline & REGIONAL-REGIONAL &,- 220 & ,087 &,- 061 & $-2,548$ & ,011 \\
\hline & REGIONAL-ESPAÑOL & , 132 & 078 &, 040 & 1,691 & ,091 \\
\hline \multirow{7}{*}{$2 \mathrm{R}^{2}=3,6 \%$} & (Constante) & ,636 & ,208 & & 3,062 & ,002 \\
\hline & ESPAÑOL-REGIONAL &,- 277 &, 100 &,- 068 & $-2,764$ &, 006 \\
\hline & REGIONAL-REGIONAL &,- 044 & ,090 &,- 012 &,- 488 & ,626 \\
\hline & REGIONAL-ESPAÑOL & ,109 & ,078 & ,033 & 1,405 & ,160 \\
\hline & CENTRO PRIVADO & ,332 &, 062 &, 141 & 5,405 &, 000 \\
\hline & ESCS & ,042 & ,026 & ,041 & 1,633 & ,103 \\
\hline & PISA CIENCIAS &,- 001 & ,000 &,- 072 & $-2,948$ & ,003 \\
\hline
\end{tabular}

Tabla 8F. CATALUÑ A

\begin{tabular}{|c|c|c|c|c|c|c|}
\hline & \multirow[t]{2}{*}{ MODELO } & \multicolumn{2}{|c|}{$\begin{array}{l}\text { COEFICIENTES NO } \\
\text { ESTANDARIZADOS }\end{array}$} & \multirow{2}{*}{$\begin{array}{c}\text { COEFICIE } \\
\text { NTES } \\
\text { TIPIFICAD } \\
\text { OS } \\
\text { BETA }\end{array}$} & \multirow[t]{2}{*}{$\mathbf{T}$} & \multirow[t]{2}{*}{ SIG. } \\
\hline & & B & $\begin{array}{c}\text { ERROR } \\
\text { TÍP. }\end{array}$ & & & \\
\hline \multirow{2}{*}{$1 \mathrm{R}^{2}=0,0$} & (Constante) & ,207 & 035 & & 5,856 & ,000 \\
\hline & REGIONAL-REGIONAL & 059 &, 050 & 025 & 1,190 & 234 \\
\hline \multirow{5}{*}{$2 \mathrm{R}^{2}=0,7 \%$} & (Constante) &,- 197 &, 185 & & $-1,065$ & 287 \\
\hline & REGIONAL-REGIONAL & ,002 & 053 & ,001 & ,042 & ,967 \\
\hline & CENTRO PRIVADO & 146 & 055 & 062 & 2,666 & ,008 \\
\hline & ESCS &, 040 & ,027 &, 037 & 1,477 &, 140 \\
\hline & PISA CIENCIAS & 000 &, 000 & 030 & 1,344 & ,179 \\
\hline
\end{tabular}

Nota: Ponderación por CA, CCAA bilingües, solo nativos de lengua doméstica española.

Fuente: Datos PISA, 2015 
Tabla 9. Determinantes del sentimiento de pertenencia a la escuela (SdPE), España*, 2015

\begin{tabular}{|c|c|c|c|c|c|c|}
\hline & \multirow[t]{2}{*}{ MODELO } & \multicolumn{2}{|c|}{$\begin{array}{c}\text { COEFICIENTES } \\
\text { NO } \\
\text { ESTANDARIZADOS } \\
\end{array}$} & \multirow{2}{*}{$\begin{array}{c}\text { COEFICIENTES } \\
\text { TIPIFICADOS } \\
\text { BETA }\end{array}$} & \multirow[t]{2}{*}{$\mathbf{T}$} & \multirow[t]{2}{*}{ SIG. } \\
\hline & & B & $\begin{array}{c}\text { ERROR } \\
\text { TÍP. }\end{array}$ & & & \\
\hline \multirow{7}{*}{$1 \mathrm{R}^{2}=1,7 \%$} & (Constante) &, 600 & ,008 & & 74,675 & 0,000 \\
\hline & BALEARES &,- 397 & 044 &,- 050 & $-8,984$ & ,000 \\
\hline & CATALUÑA &,- 370 &, 018 &,- 115 & $-20,359$ &, 000 \\
\hline & GALICIA &,- 344 & ,029 &,- 066 & $-11,797$ &, 000 \\
\hline & NAVARRA &,- 030 &, 052 &,- 003 &,- 576 &, 564 \\
\hline & CA VASCA &,- 098 & ,031 &,- 018 & $-3,158$ & ,002 \\
\hline & C. VALENCIANA &,- 063 & ,022 &,- 016 & $-2,867$ & ,004 \\
\hline \multirow{8}{*}{$2 \mathrm{R}^{2}=2 \%$} & (Constante) &, 600 & ,008 & & 74,807 & 0,000 \\
\hline & BALEARES &,- 105 &, 052 &,- 013 & $-2,032$ &, 042 \\
\hline & CATALUÑA &,- 038 &, 036 &,- 012 & $-1,065$ & ,287 \\
\hline & GALICIA &,- 120 &, 036 &,- 023 & $-3,350$ &, 001 \\
\hline & NAVARRA &, 059 &, 053 &, 006 & 1,117 & ,264 \\
\hline & CA VASCA & 131 &, 037 &, 024 & 3,488 &, 000 \\
\hline & C. VALENCIANA & ,007 & 023 & ,002 & 297 &, 767 \\
\hline & LENGUA ESCOLAR REGIONAL &,- 332 & 031 &,- 127 & $-10,729$ & ,000 \\
\hline \multirow{12}{*}{$3 \mathrm{R}^{2}=2,1 \%$} & (Constante) &, 600 & ,008 & & 74,843 & 0,000 \\
\hline & BALEARES &,- 105 &, 060 &,- 013 & $-1,738$ &, 082 \\
\hline & CATALUÑA &,- 037 &, 050 &,- 012 &,- 749 & ,454 \\
\hline & GALICIA &, 030 &, 050 & ,006 &, 594 &, 553 \\
\hline & NAVARRA &, 059 &, 054 &, 006 & 1,104 &, 270 \\
\hline & CA VASCA &,- 001 & ,046 &, 000 &,- 031 & ,976 \\
\hline & C. VALENCIANA &,- 002 & ,024 & 000 &,- 064 & ,949 \\
\hline & LENGUA ESCOLAR REGIONAL &,- 332 & ,047 &,- 127 & $-7,124$ &, 000 \\
\hline & ESPAÑOL-GALLEGO &,- 252 & ,083 &,- 029 & $-3,026$ &, 002 \\
\hline & GALLEGO-GALLEGO &,- 190 &, 083 &,- 022 & $-2,287$ &, 022 \\
\hline & ESPAÑOL-VASCUENCE & ,263 &, 066 &, 034 & 3,957 &, 000 \\
\hline & VALENCIANO-VALENCIANO & ,072 & ,076 & ,007 & ,950 & ,342 \\
\hline \multirow{15}{*}{$4 \mathrm{R}^{2}=2,7 \%$} & (Constante) &, 727 &, 041 & & 17,544 &, 000 \\
\hline & BALEARES &,- 134 &, 060 &,- 017 & $-2,223$ &, 026 \\
\hline & CATALUÑA &,- 088 & ,050 &,- 028 & $-1,754$ &, 080 \\
\hline & GALICIA &, 004 &, 050 & ,001 &, 073 & ,942 \\
\hline & NAVARRA &, 025 & ,054 & ,003 & 469 & ,639 \\
\hline & CA VASCA &,- 071 & 047 &,- 013 & $-1,515$ &, 130 \\
\hline & C. VALENCIANA &,- 013 & ,024 &,- 003 &,- 551 &, 581 \\
\hline & LENGUA ESCOLAR REGIONAL &,- 306 & ,047 &,- 117 & $-6,532$ &, 000 \\
\hline & ESPAÑOL-GALLEGO &,- 247 & ,083 &,- 028 & $-2,981$ & ,003 \\
\hline & GALLEGO-GALLEGO &,- 142 & ,083 &,- 016 & $-1,717$ &, 086 \\
\hline & ESPAÑOL-VASCUENCE &, 280 & ,066 & 036 & 4,223 &, 000 \\
\hline & VALENCIANO-VALENCIANO & ,087 & ,076 & ,008 & 1,142 & ,253 \\
\hline & CENTRO PRIVADO & ,056 &, 014 &, 023 & 3,902 &, 000 \\
\hline & ESCS & ,068 & ,006 & 070 & 11,220 &, 000 \\
\hline & PISA CIENCIAS & ,000 & ,000 &,- 017 & $-2,783$ &, 005 \\
\hline
\end{tabular}

*CCAA bilingües separadas.

Nota:Ponderación nacional, solo nativos de lengua doméstica española.

Fuente: Datos PISA, 2015 
Tabla A1. Estadísticos descriptivos y correlaciones, variables en la regresión de la tabla 6, España, 2015.

\begin{tabular}{|c|c|c|c|c|c|c|c|c|c|c|c|}
\hline & \multicolumn{3}{|c|}{ DESCRIPTIVOS } & \multicolumn{8}{|c|}{ CORRELACIONES } \\
\hline $\begin{array}{l}\text { VARIABLES } \\
\text { EN LA } \\
\text { REGRESIÓN }\end{array}$ & MEDIA & $\begin{array}{l}\text { DESVIACIÓN } \\
\text { TÍPICA }\end{array}$ & $\mathbf{N}$ & 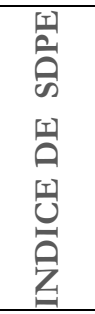 & 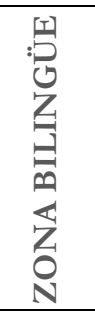 & 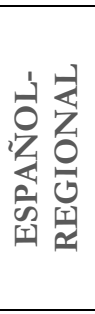 & 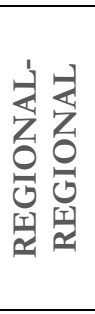 & 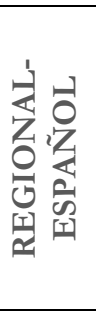 & 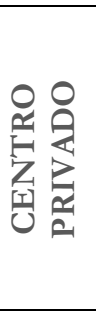 & 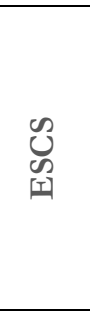 & 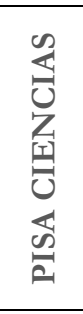 \\
\hline $\begin{array}{l}\text { INDICE DE } \\
\text { SdPE }\end{array}$ &, 507438 & 1,1480210 & 32285 & 1,000 &,- 104 &,- 095 &,- 084 & ,021 &, 047 &, 062 &,- 002 \\
\hline $\begin{array}{l}\text { ZONA } \\
\text { BILINGÜE }\end{array}$ & ,3788 & ,48510 & 32285 &,- 104 & 1,000 &, 511 & ,478 & ,171 &, 080 &, 088 & ,077 \\
\hline $\begin{array}{l}\text { ESPAÑOL- } \\
\text { REGIONAL }\end{array}$ & ,1374 & ,34432 & 32285 &,- 095 &, 511 & 1,000 &,- 149 &,- 053 & -,012 &,- 003 & ,004 \\
\hline $\begin{array}{l}\text { REGIONAL- } \\
\text { REGIONAL }\end{array}$ & ,1223 & ,32762 & 32285 &,- 084 & ,478 &,- 149 & 1,000 &,- 050 &, 000 &, 104 & ,114 \\
\hline $\begin{array}{l}\text { REGIONAL- } \\
\text { ESPAÑOL }\end{array}$ & ,0175 & ,13117 & 32285 & ,021 & ,171 &,- 053 &,- 050 & 1,000 &, 057 & ,002 & ,006 \\
\hline $\begin{array}{l}\text { CENTRO } \\
\text { PRIVADO }\end{array}$ & 1,3578 & ,47936 & 32285 & ,047 & ,080 &,- 012 & ,000 &, 057 & 1,000 &, 359 & , 156 \\
\hline ESCS &,- 442077 & 1,1806830 & 32285 & ,062 & ,088 &,- 003 & 104 & ,002 & ,359 & 1,000 & 363 \\
\hline $\begin{array}{l}\text { PISA } \\
\text { CIENCIAS }\end{array}$ & 500,39538 & 85,929668 & 32285 &,- 002 & 077 &, 004 & ,114 & ,006 & ,156 &, 363 & 1,000 \\
\hline
\end{tabular}

Nota:Ponderación nacional, solo nativos de lengua doméstica española.

Fuente: Datos PISA, 2015 